\title{
To the Fifth Dimension and Back
}

\author{
Raman Sundrum* \\ Department of Physics and Astronomy \\ The Johns Hopkins University \\ 3400 North Charles Street \\ Baltimore, MD 21218, USA
}

\begin{abstract}
Introductory lectures on Extra Dimensions delivered at TASI 2004.
\end{abstract}

\section{Introduction}

There are several significant motivations for studying field theory in higher dimensions: (i) We are explorers of spacetime structure, and extra spatial dimensions give rise to one of the few possible extensions of relativistic spacetime symmetry. (ii) Extra dimensions are required in string theory as the price for taming the bad high energy behavior of quantum gravity within a weakly coupled framework. (iii) Extra dimensions give rise to qualitatively interesting mechanisms within effective field theory that may play key roles in our understanding of Nature. (iv) Extra dimensions can be a type of "emergent" phenomenon, as best illustrated by the famous AdS/CFT correspondence.

*sundrum@pha.jhu.edu 
These lectures are intended to provide an introduction, not to the many attempts at realistic extra-dimensional model-building, but rather to central qualitative extra-dimensional mechanisms. It is of course hoped that by learning these mechanisms in their simplest and most isolated forms, the reader is well-equipped to work through more realistic incarnations and combinations, in the literature, or better yet, as products of their own invention. (Indeed, to really digest these lectures, the reader must use them to understand some particle physics models and issues. The other TASI lectures are a good place to start.) When any of the examples in the lectures yields a cartoon of the real world, or a cartoon solution to real world problems, I point this out.

The lectures are organized as follows. Section 2 gives the basic language for dealing with field theory in the presence of extra dimensions, "compactified" in order to hide them at low energies. It is also shown how particles of different spins in four dimensions can be unified within a single higherdimensional field. Section 3 illustrates the "chirality problem" associated with fermions in higher dimensions. Section 4 illustrates the emergence of light scalars from higher dimensional theories without fundamental scalars, computes quantum corrections to the scalar mass (potential), and assesses how natural these light scalars are. Section 5 describes how extra dimensional boundaries (and boundary conditions) can be derived from extra dimensional spaces without boundary, by the procedure of "orbifolding". It is shown how the chirality problem can thereby be solved. The localization of some fields to the boundaries is illustrated. Section 6 describes the matching of the higher dimensional couplings to the effective four-dimensional long-distance couplings. In Section 7, the issue of non-renormalizability of higher-dimensional field theory is discussed and the scale at which a UV completion is required is identified. Higher-dimensional General Relativity is discussed in Section 8 , in partiucular the emergence of extra gauge fields at low energies as well as scalar "radion" fields (or "moduli") describing massless fluctuations in the extra-dimensional geometry. Section 9 illustrates how moduli may be stabilized to fix the extra-dimensional geometry at low energies. Section 10 describes the unsolved Cosmological Constant Problem as well as the less problematic issue of having a higher-dimensional cosmological constant. Section 10 shows that a higher dimensional cosmological constant leads to "warped" compactifications, as well as the phenomenon of "gravity localization". Section 11 shows that strongly warped compactifications naturally lead to hierarchies in the mass scales appearing in the low energy effective 
four-dimensional description. Section 12 shows that when warped hierarchies are used to generate the Planck/weak-scale hierarchy, the extra-dimensional graviton excitations are much more strongly coupled to matter than the massless graviton of nature, making them observable at colliders. Section 13 shows how flavor hierarchies and flavor protection can arise naturally in warped compactification, following from a study of higher-dimensional fermions. Section 14 studies features of gauge theory, including the emergence of light scalars, in warped compactifications.

The TASI lectures of Ref. [1] and Ref. [2], and the Cargese lectures of Ref. [3], while overlapping with the present lectures, also contain complementary topics and discussion. The central qualitative omissions in the present lectures are supersymmetry, which can combine with extra dimensions in interesting ways (see the TASI lectures of Refs. [1] and [4]), a longer discussion of the connection of extra dimensions to string theory [5] [6], a discussion of fluctuating "branes" (see Refs. [1] and [3]), and the (very illuminating) AdS/CFT correspondence between some warped extra-dimensional theories and some purely four-dimensional theories with strong dynamics [7] [8] 9]. Phenomenologically, there is no discussion of the "Large Extra Dimensions" scenario [10, although these lectures will equip the reader to easily understand it.

The references included are meant to be useful and to act as gateways to the broader literature. They are not intended to be a complete set. I have taken moderate pains to get incidental numbers right in the notes, but I am fallible. I have taken greater pains to ensure that important numbers, such as exponents, are correct.

\section{Compactification and Spin Unification}

Let us start by considering $S U(2)$ Yang-Mills (YM) theory in five-dimensional (5D) Minkowski spacetime, ${ }^{1}$ in particular all dimensions being infinite in size,

$$
\begin{aligned}
S & =\operatorname{Tr} \int d^{4} x \int d x_{5}\left\{-\frac{1}{4} F_{M N} F^{M N}\right\} \\
& =\operatorname{Tr} \int d^{4} x \int d x_{5}\left\{-\frac{1}{4} F_{\mu \nu} F^{\mu \nu}-\frac{1}{2} F_{\mu 5} F^{\mu 5}\right\},
\end{aligned}
$$

\footnotetext{
${ }^{1}$ Our metric signature convention throughout these lectures is $(+-\ldots-)$.
} 
where $M, N=0,1,2,3,5$ are $5 \mathrm{D}$ indices, while $\mu, \nu=0,1,2,3$ are $4 \mathrm{D}$ indices. We use matrix notation for $S U(2)$ so that the gauge field is $A_{M} \equiv A_{M}^{a} \tau_{a}$, where $\tau_{a}$ are the isospin Pauli matrices. We will study this theory in an axial gauge, $A_{5}=0$. To see that this is a legitimate gauge, imagine that $A_{M}$ is in a general gauge and consider a gauge transformation,

$$
A_{M}^{\prime} \equiv \frac{i}{g} \Omega^{-1} D_{M} \Omega, \Omega\left(x^{\mu}, x_{5}\right) \in S U(2)
$$

where $g$ is the gauge coupling. It is always possible to find $\Omega\left(x, x_{5}\right)$, such that $A_{5}^{\prime}=0$.

Ex. Check that this happens for $\Omega\left(x, x_{5}\right)=\mathcal{P} e^{i g \int_{0}^{x_{5}} d x_{5}^{\prime} A_{5}\left(x, x_{5}^{\prime}\right)}$, where $\mathcal{P}$ represents the path-ordering of the exponential.

Ex. Check that in this gauge,

$$
S=\operatorname{Tr} \int d^{4} x \int d x_{5}\left\{-\frac{1}{4} F_{\mu \nu} F^{\mu \nu}+\frac{1}{2}\left(\partial_{5} A_{\mu}\right)^{2}\right\} .
$$

Let us now compactify the fifth dimension to a circle, so that $x_{5} \equiv R \phi$, where $R$ is the radius of the circle and $\phi$ is an angular coordinate $-\pi \leq \phi \leq \pi$. See Fig. 1. We can Fourier expand the gauge field in this coordinate,

$$
A_{\mu}\left(x^{\mu}, \phi\right)=A_{\mu}^{(0)}(x)+\sum_{n=1}^{\infty}\left(A_{\mu}^{(n)}(x) e^{i n \phi}+\text { h.c. }\right) .
$$

But now we can no longer go to axial gauge; in general our $\Omega$ above will not be $2 \pi$-periodic. The best we can do is go to an "almost axial" gauge where $A_{5}$ is $\phi$-independent, $A_{5}(x, \phi) \equiv A_{5}^{(0)}(x)$, where the action can be written

$$
\begin{aligned}
S= & \operatorname{Tr} \int d^{4} x \int_{-\pi}^{\pi} d \phi R\left\{-\frac{1}{4} F_{\mu \nu} F^{\mu \nu}+\frac{1}{2}\left(D_{\mu} A_{5}^{(0)}\right)^{2}+\frac{1}{2}\left(\partial_{5} A_{\mu}\right)^{2}\right\} \\
= & 2 \pi R \operatorname{Tr} \int d^{4} x\left\{-\frac{1}{2}\left(\partial_{\mu} A_{\nu}^{(0)}-\partial_{\nu} A_{\mu}^{(0)}\right)^{2}+\frac{1}{2}\left(\partial_{\mu} A_{5}^{(0)}\right)^{2}\right. \\
& \left.+\sum_{n=1}^{\infty}\left[-\frac{1}{2}\left|\partial_{\mu} A_{\nu}^{(n)}-\partial_{\nu} A_{\mu}^{(n)}\right|^{2}+\frac{n^{2}}{R^{2}}\left|A_{\mu}^{(n)}\right|^{2}\right]+\mathcal{O}\left(A^{3}\right)\right\},
\end{aligned}
$$

showing that the 5D theory is equivalent to a 4D theory with an infinite tower of $4 \mathrm{D}$ fields, with masses, $m_{n}^{2}=n^{2} / R^{2}$. This rewriting of $5 \mathrm{D}$ compactified physics is called the Kaluza-Klein (KK) decomposition. 


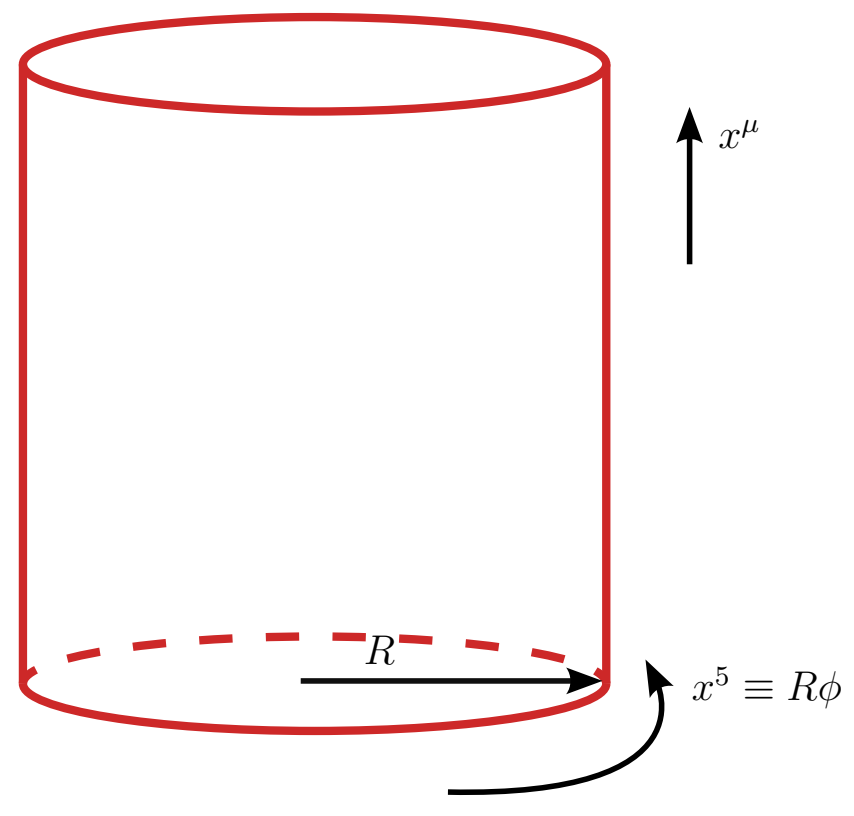

Figure 1: A 5D hypercylindrical spacetime

Ex. Show that if $A_{M}$ is in a general gauge it can be brought to almost axial gauge via the (periodic) gauge transformation

$$
\Omega(x, \phi) \equiv \mathcal{P} e^{i g \int_{0}^{\phi} d \phi^{\prime} R A_{5}\left(x, \phi^{\prime}\right)} e^{-i g A_{5}^{(0)}(x) \phi} .
$$

Note that the sum over $n$ of the fields in any interaction term must be zero since this is just conservation of fifth dimensional momentum, where for convenience we define the complex conjugate modes, $A_{\mu}^{(n) *}$, to be the modes corresponding to $-n$. In this way a spacetime symmetry and conservation law appears as an internal symmetry in the 4D KK decomposition, with internal charges, $n$.

Since all of the $n \neq 0$ modes have $4 \mathrm{D}$ masses, we can write a $4 \mathrm{D}$ effective theory valid below $1 / R$ involving just the light $A^{(0)}$ modes. Tree level matching yields

$$
S_{\text {eff }} \underset{E \ll \frac{1}{R}}{\sim} 2 \pi R \operatorname{Tr} \int d^{4} x\left\{-\frac{1}{4} F_{\mu \nu}^{(0)} F^{(0) \mu \nu}+\frac{1}{2}\left(D_{\mu} A_{5}^{(0)}\right)^{2}\right\} .
$$

The leading (renormalizable) non-linear interactions follow entirely from the $4 \mathrm{D}$ gauge invariance which survives almost axial gauge fixing. We have a 


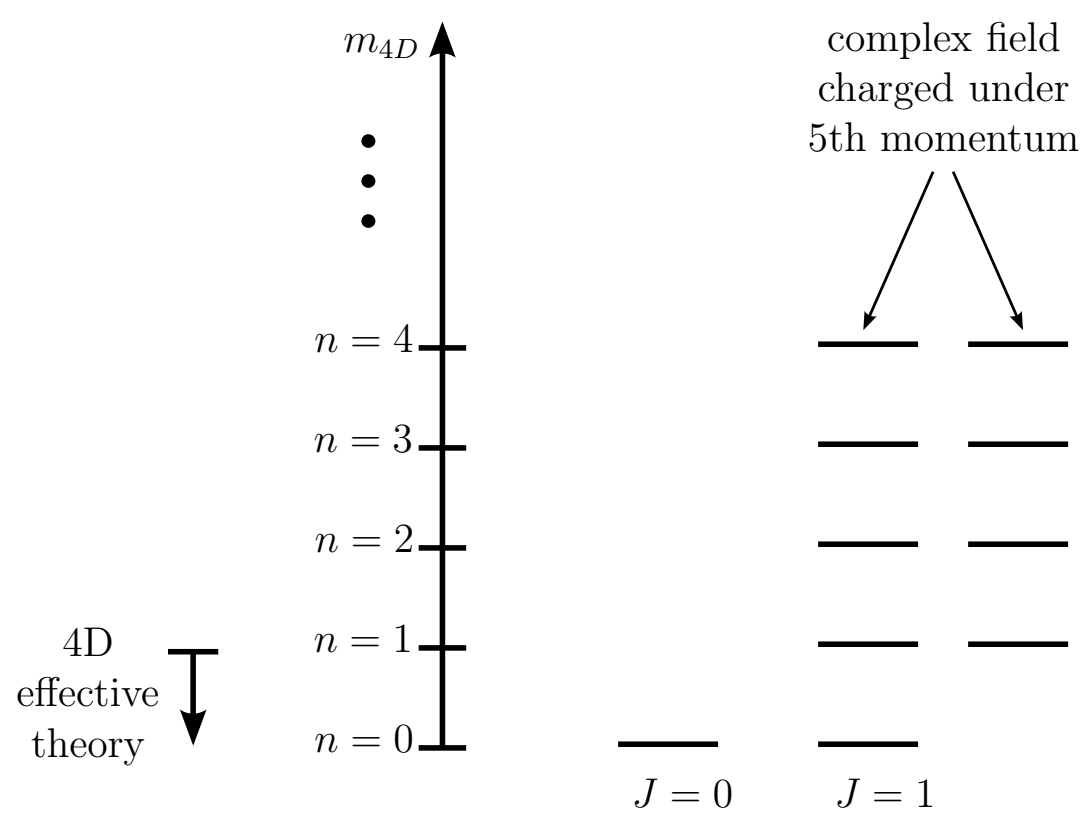

Figure 2: 4D KK spectrum of 5D gauge field

theory of a 4D gauge field and gauge-charged 4D scalar, unified in their higher-dimensional origins. This unification is hidden along with the extra dimension at low energies, but for $E \gg 1 / R$ the tell-tale "Kaluza-Klein" (KK) excitations $A^{(n)}$ are accessible, and the full story can be reconstructed in principle.

Ex. Check that almost axial gauge is preserved by 4D gauge transformations, $\Omega(x)$ (independent of $\phi$ ).

Our results are summarized in Fig. 2.

\section{5D Fermions and the Chirality Problem}

To proceed we need a representation of the 5D Clifford algebra, $\left\{\Gamma_{M}, \Gamma_{N}\right\}=$ $2 \eta_{M N}$. This is straightforwardly provided by

$$
\Gamma_{\mu} \equiv \gamma_{\mu}, \quad \Gamma_{5} \equiv-i \gamma_{5}
$$


where the $\gamma$ 's are the familiar 4D Dirac matrices. Therefore, 5D fermions are necessarily 4-component spinors. We decompose them as

$$
\Psi_{\alpha}(x, \phi)=\sum_{n=-\infty}^{\infty} \Psi_{\alpha}^{(n)}(x) e^{i n \phi}
$$

Plugging this into the 5D Dirac action gives

$$
\begin{aligned}
S_{\Psi} & =\int d^{4} x \int d x_{5} \bar{\Psi}\left(i D_{M} \Gamma^{M}-m\right) \Psi \\
& =\int d^{4} x \int d x_{5} \bar{\Psi}\left(i D_{\mu} \gamma^{\mu}-m\right) \Psi-\bar{\Psi} \gamma_{5} \partial_{5} \Psi+i g \bar{\Psi} A_{5} \gamma_{5} \Psi \\
& =2 \pi R \int d^{4} x \sum_{n=-\infty}^{\infty} \bar{\Psi}^{(n)}\left(i \gamma^{\mu} \partial_{\mu}-m-i \frac{n}{R} \gamma_{5}\right) \Psi^{(n)}+\mathcal{O}(\bar{\Psi} A \Psi) .
\end{aligned}
$$

We see that we get a tower of 4D Dirac fermions labelled by integer $n$ (no longer positive), with physical masses,

$$
m_{\mathrm{phys}}^{2}=m^{2}+\frac{n^{2}}{R^{2}}
$$

For small $m$, this is illustrated in Fig. 3. These fermions are coupled to the gauge field KK tower, again with all interactions conserving 5D momentum, the sum over $n$ of all $4 \mathrm{D}$ fields in an interaction adding up to zero.

At low energies, $E \ll 1 / R$, we can again get a $4 \mathrm{D}$ effective action for the light $n=0$ modes,

$$
S_{\text {eff }} \underset{\substack{E \ll \frac{1}{R} \\ m \ll \frac{1}{R}}}{=} 2 \pi R \int d^{4} x\left\{\bar{\Psi}^{(0)}\left(i \gamma^{\mu} D_{\mu}-m\right) \Psi^{(0)}+i g \bar{\Psi}^{(0)} \gamma_{5} A_{5}^{(0)} \Psi^{(0)}\right\},
$$

where the covariant derivative contains only the gauge field $A_{\mu}^{(0)}$. Note that we also have a Yukawa coupling to the $4 \mathrm{D}$ scalar, $A_{5}^{(0)}$, of the same strength as the gauge coupling, so-called gauge-Yukawa unification. The idea that the Higgs particle may originate from extra-dimensional components of gauge fields was first discussed in Refs. [11].

An unattractive feature in this cartoon of the real world, emerging below $1 / R$, is that the necessity of having Dirac 4-component spinor representations 


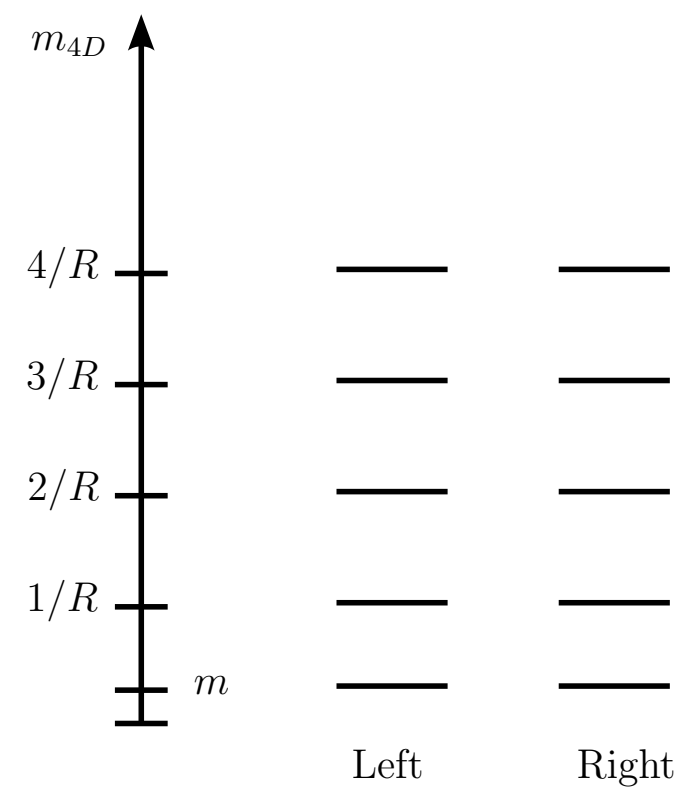

Figure 3: 4D KK spectrum of 5D fermion

of 5D Lorentz invariance has resulted in having 4-component non-chiral 4D fermion zero-modes. The Standard Model is however famously a theory of chiral Weyl 2-component fermions. Even as a cartoon this looks worrying. This general problem in theories of higher dimensions is called the "chirality problem" and we will return to deal with it later.

\section{Light Scalar Quantum Corrections}

Given that light scalars are unnatural in (non-supersymmetric) quantum field theories, it is rather surprising to see a massless $4 \mathrm{D}$ scalar, $A_{5}^{(0)}$, emerge from higher dimensions. Of course, we should consider quantum corrections to our classical story and see what happens to the scalar mass. From a purely 4D effective field theory viewpoint we would expect there to be large divergent corrections to the scalar mass coming from its gauge and Yukawa couplings, from diagrams such as Fig. 4,

$$
\delta m_{\mathrm{scalar}}^{2} \sim \frac{g_{4}^{2}}{16 \pi^{2}} \Lambda_{\mathrm{UV}}^{2}
$$




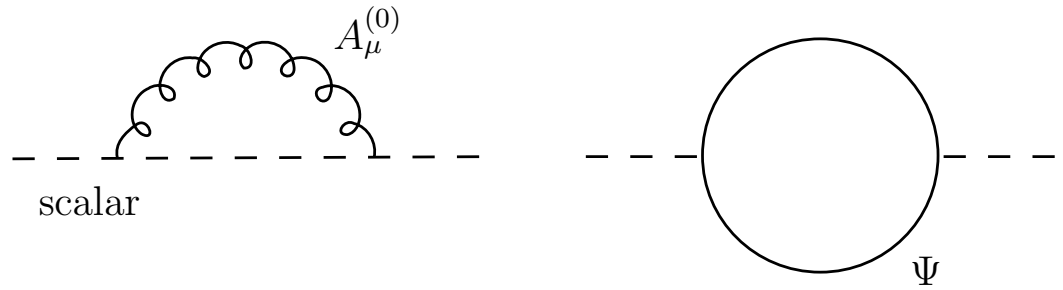

Figure 4: Some quantum corrections to 4D scalar self energy

suggesting that the scalar is naturally very heavy. But from the $5 \mathrm{D}$ viewpoint $A_{5}$ is massless because it is part of a $5 \mathrm{D}$ gauge field, whose mass is protected by $5 \mathrm{D}$ gauge invariance. So the question is which viewpoint is correct?

To find out let us first compute the 1-fermion-loop effective potential for $A_{5}^{(0)}\left[12\right.$. For this purpose we treat $a \equiv g A_{5}^{(0)}$ as completely constant, and $A_{\mu}=0$. Then,

$$
S_{\Psi}=2 \pi R \int d^{4} x \sum_{n} \bar{\Psi}^{(n)}(x)\left[i \not \partial-m-i\left(\frac{n}{R}-a\right) \gamma_{5}\right] \Psi^{(n)}(x),
$$

where

$$
\not \supset \equiv \gamma^{\mu} \partial_{\mu}
$$

Since $a$ is constant,

$$
S_{\Psi}=2 \pi R \int \frac{d^{4} p}{(2 \pi)^{4}} \sum_{n} \bar{\Psi}^{(n)}(p)\left[\not p-m-i\left(\frac{n}{R}-a\right) \gamma_{5}\right] \Psi^{(n)}(p) .
$$

After Wick rotating, this gives

$$
S_{\Psi}^{\mathrm{E}}=\sum_{n} 2 \pi R \int \frac{d^{4} p}{(2 \pi)^{4}} \bar{\Psi}^{(n)}(p)\left[\not p+i m+\left(\frac{n}{R}-a\right) \gamma_{5}\right] \Psi^{(n)}(p) .
$$

Integrating out the fermions by straightforward Gaussian Grassman integration,

$$
\begin{aligned}
e^{-V_{\mathrm{eff}}} & =\prod_{p, n} \operatorname{det}\left[\not p+i m+\left(\frac{n}{R}-a\right) \gamma_{5}\right] \\
& =\exp \left\{\sum_{n} \int \frac{d^{4} p}{(2 \pi)^{4}} \operatorname{tr} \ln \left[\not p+i m+\left(\frac{n}{R}-a\right) \gamma_{5}\right]\right\} .
\end{aligned}
$$


From now on, I will simplify slightly by considering a $U(1)$ gauge group rather than $S U(2)$. All subtleties will come from finite $R$, so we focus on

$$
\begin{aligned}
\frac{\partial V_{\text {eff }}}{\partial R} & =-\sum_{n} \int \frac{d^{4} p}{(2 \pi)^{4}} \operatorname{tr}\left[-\frac{n}{R^{2}} \gamma_{5} \frac{1}{\not p+i m+\left(\frac{n}{R}-a\right) \gamma_{5}}\right] \\
& =\sum_{n} \int \frac{d^{4} p}{(2 \pi)^{4}} \operatorname{tr}\left[\frac{n}{R^{2}} \gamma_{5} \frac{\not p-i m+\left(\frac{n}{R}-a\right) \gamma_{5}}{p^{2}+\left(\frac{n}{R}-a\right)^{2}+m^{2}}\right] \\
& =\sum_{n} \int \frac{d^{4} p}{(2 \pi)^{4}} \frac{4 n(n-a)}{p^{2}+(n-a)^{2}+m^{2}},
\end{aligned}
$$

where we have gone to $R \equiv 1$ units in the last line.

Naively, this integal and sum over $n$ is quintically divergent! So let us carefully regulate the calculation by adding Pauli-Villars fields, in a 5D gaugeinvariant manner. These fields have the same quantum numbers as $\Psi$, but have cut-off size masses $m \rightarrow \Lambda_{\mathrm{UV}}$, some with Bose rather than Fermi statistics. Thereby,

$$
\frac{\partial V_{\mathrm{eff}}}{\partial R}=\sum_{n} \int \frac{d^{4} p}{(2 \pi)^{4}}\left(\frac{4 n(n-a)}{p^{2}+(n-a)^{2}+m^{2}}+\begin{array}{c}
\text { regulator } \\
\text { terms }
\end{array}\right)<\infty .
$$

The regulator terms resemble the physical term except for having cutoff size masses and with signs (determined by the statistics of the regulator field) chosen in such a way that the entire expression converges. The big trick for doing the sum on $n$ is to replace it by a contour integral,

$$
\frac{\partial V_{\mathrm{eff}}}{\partial R}=\int \frac{d^{4} p}{(2 \pi)^{4}} \oint_{C} d z \frac{1}{e^{2 \pi i z}-1}\left(\frac{4 z(z-a)}{p^{2}+(z-a)^{2}+m^{2}}+\text { Reg. }\right),
$$

where the contour is shown in Fig. 5, following from the simple poles of the factor $1 /\left(e^{2 \pi i z}-1\right)$ and from the residue theorem. The semi-circles at infinity needed to have a closed contour are irrelevant because the integrand vanishes rapidly enough there, precisely because of the addition of the regulator terms. We can deform the contour to that shown in Fig. 6 without encountering any singularities of the integrand, so that by the residue theorem,

$$
\begin{aligned}
\frac{\partial V_{\mathrm{eff}}}{\partial R}=-4 \pi i \int \frac{d^{4} p}{(2 \pi)^{4}}\left[\frac{a+i \sqrt{p^{2}+m^{2}}}{e^{2 \pi i a} e^{-2 \pi \sqrt{p^{2}+m^{2}}}-1}\right. \\
\left.+\frac{a-i \sqrt{p^{2}+m^{2}}}{e^{2 \pi i a} e^{2 \pi \sqrt{p^{2}+m^{2}}}-1}+\text { Reg. }\right] .
\end{aligned}
$$




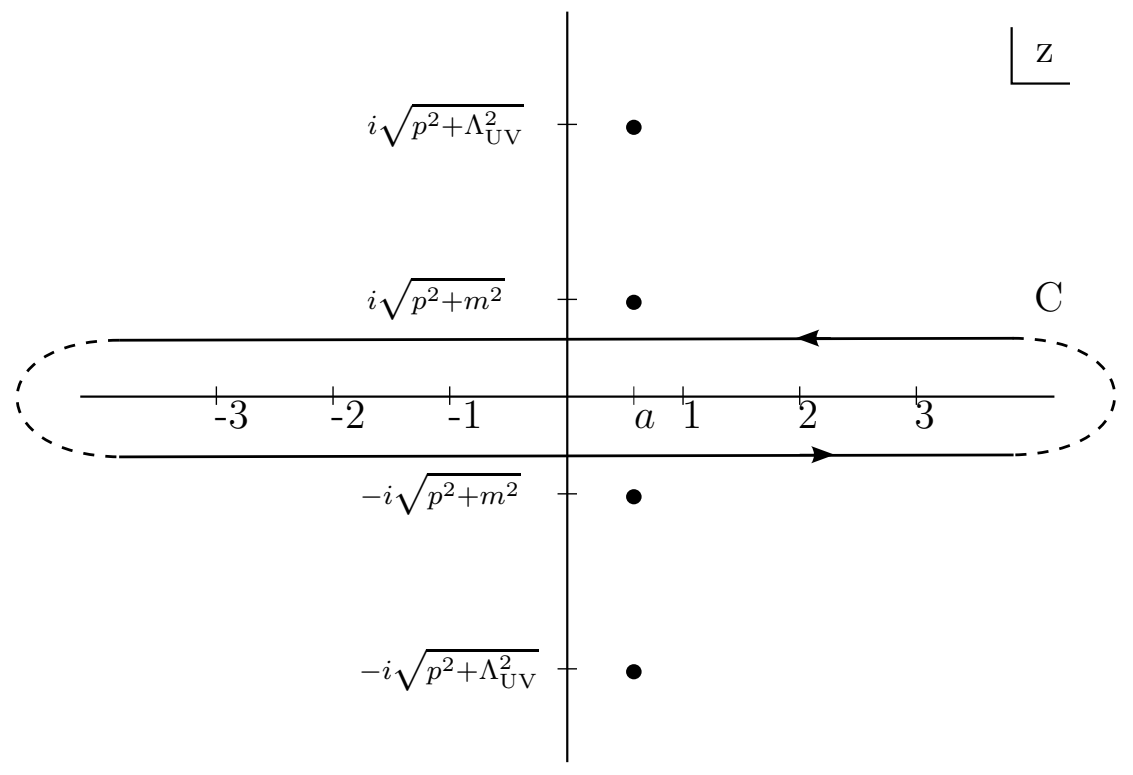

Figure 5: Contour for turning KK sum into integral

We can also write this as

$$
\begin{aligned}
\frac{\partial V_{\mathrm{eff}}}{\partial R}=4 \pi \int \frac{d^{4} p}{(2 \pi)^{4}}[ & \overbrace{\frac{\sqrt{p^{2}+m^{2}}-i a}{e^{2 \pi i a} e^{-2 \pi \sqrt{p^{2}+m^{2}}}-1}-\frac{\sqrt{p^{2}+m^{2}}+i a}{e^{2 \pi i a} e^{2 \pi \sqrt{p^{2}+m^{2}}}-1}} \\
& +\left(\sqrt{p^{2}+m^{2}}-i a\right)\left(\frac{e^{2 \pi i a} e^{-2 \pi \sqrt{p^{2}+m^{2}}}-\overbrace{1}}{e^{2 \pi i a} e^{-2 \pi \sqrt{p^{2}+m^{2}}}-1}\right) \\
& \left.-\left(\sqrt{p^{2}+m^{2}}-i a\right)+\text { Reg. }\right],
\end{aligned}
$$

where we have just added and subtracted the same quantity in the last two terms (not counting the regulator terms). Note that the overbraced terms cancel out, leaving

$$
\begin{aligned}
\frac{\partial V_{\mathrm{eff}}}{\partial R}=4 \pi \int \frac{d^{4} p}{(2 \pi)^{4}} & {\left[\left(-\frac{\sqrt{p^{2}+m^{2}}-i a}{e^{-2 \pi i a R} e^{2 \pi R \sqrt{p^{2}+m^{2}}}-1}\right)+\right.\text { c.c. }} \\
- & \left.\left(\sqrt{p^{2}+m^{2}}-i a\right)\right]+ \text { Reg. }
\end{aligned}
$$




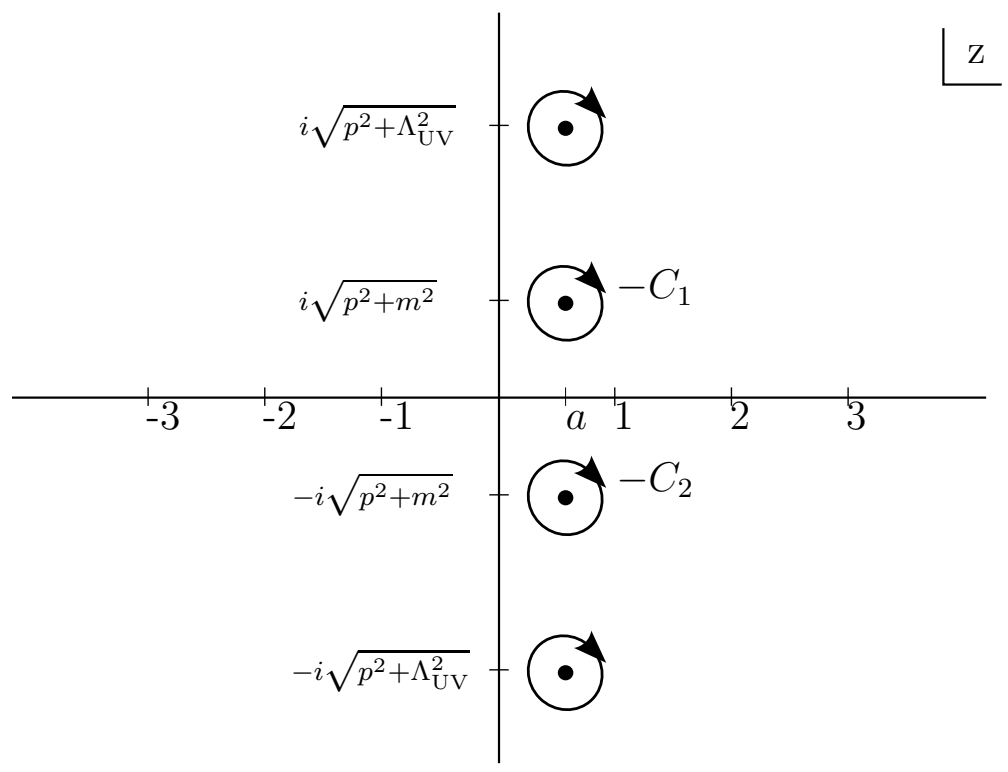

Figure 6: Deformed contour for KK sum

where we have put back $R$ explicitly, by dimensional analysis.

Now let us integrate with respect to $R$,

$$
\begin{aligned}
V_{\mathrm{eff}}=\int \frac{d^{4} p}{(2 \pi)^{4}}\left\{-4 \operatorname{Re} \ln \left(1-e^{-2 \pi R \sqrt{p^{2}+m^{2}}} e^{2 \pi i a R}\right)\right. \\
\left.-4 \pi R\left(\sqrt{p^{2}+m^{2}}-i a\right)\right\}+\operatorname{Reg} . \\
+ \text { irrelevant const. }
\end{aligned}
$$

In the $R \rightarrow \infty$ limit, $V_{\text {eff }}$ must be independent of $a$ since certainly all potential terms for gauge fields vanish by gauge invariance as usual. This yields the identity,

$$
V_{\mathrm{eff}} \underset{R \rightarrow \infty}{\longrightarrow}-4 \pi R \int \frac{d^{4} p}{(2 \pi)^{4}}\left(\sqrt{p^{2}+m^{2}}-i a\right)+\text { Reg. } \equiv \Lambda R,
$$

where $\Lambda$ is a constant independent of $a$ and $R$.

Ex. Directly show the cancellation of $a$-dependence in the right hand side of eq. (4.14) by carefully writing out the regulator terms. 


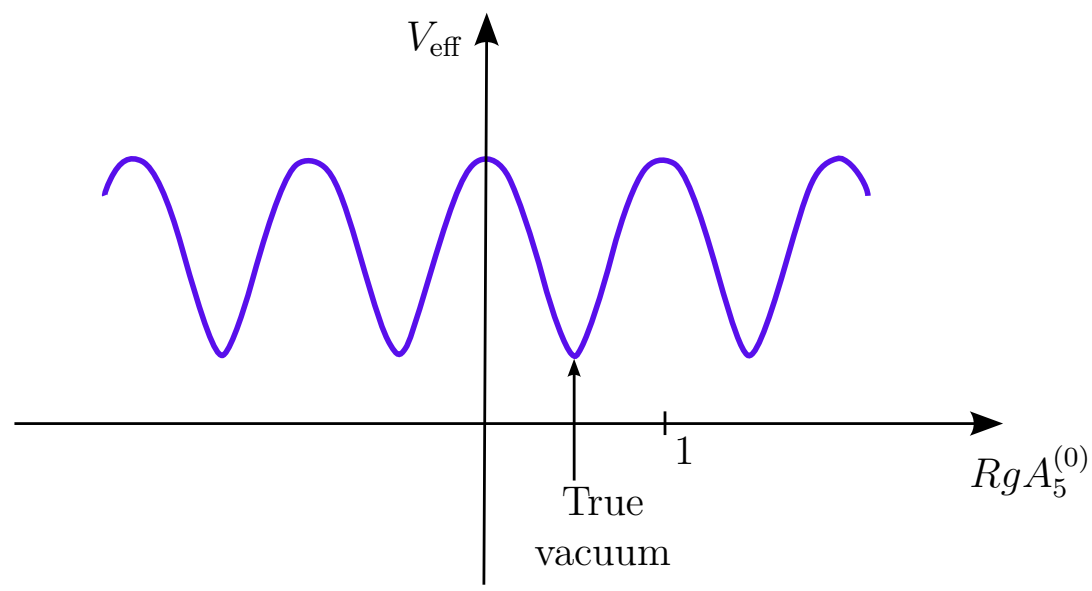

Figure 7: Quantum effective potential for $A_{5}^{(0)}$. (The potential is not exactly sinusoidal, but it is periodic.)

Using this identity in eq. (4.13) yields

$$
V_{\text {eff }}=\Lambda R-4 \int \frac{d^{4} p}{(2 \pi)^{4}} \operatorname{Re} \ln \left(1-e^{-2 \pi R \sqrt{p^{2}+m^{2}}} e^{2 \pi i a R}\right)+\text { Reg. }
$$

This formula has some remarkable properties. The first term is indeed highly cutoff dependent, but it does not depend on $a$. The integrand of the second term behaves as $e^{-2 \pi R p}$ for large $p$ and therefore the $p$ integrals converge. The regulator terms are suppressed by $e^{-2 \pi R \Lambda_{\mathrm{UV}}}$ factors and can be completely neglected for $\Lambda_{\mathrm{UV}} R \gg 1$ (or more formally, for $\Lambda_{U V} \rightarrow \infty$ ). We therefore drop the $a$-dependent regulator terms from now on.

Finally, combining complex exponentials we arrive at our final result,

$$
\begin{aligned}
V_{\mathrm{eff}}=\Lambda R-2 \int \frac{d^{4} p}{(2 \pi)^{4}} \ln & \left(1+e^{-4 \pi R \sqrt{p^{2}+m^{2}}}\right. \\
& \left.-2 e^{-2 \pi R \sqrt{p^{2}+m^{2}}} \cos \left(2 \pi R g A_{5}^{(0)}\right)\right),
\end{aligned}
$$

which is illustrated in Fig. 7 . For small $A_{5}^{(0)}$, this can be approximated, 


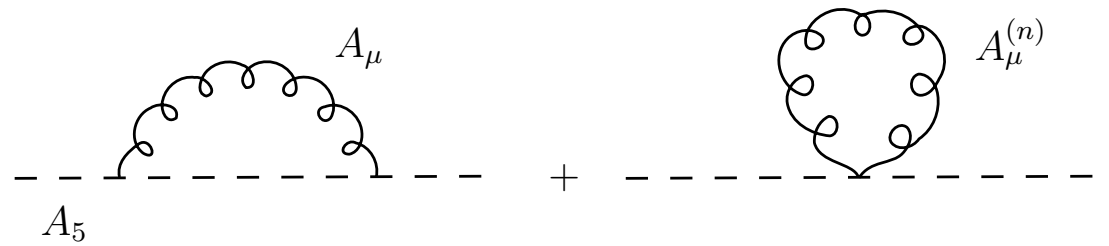

Figure 8: Examples of gauge loop contribution to $A_{5}$ potential

$$
\begin{gathered}
V_{\text {eff }} \sim \Lambda R+\int \frac{d^{4} p}{(2 \pi)^{4}}\left\{-4 \ln \left(1-e^{-2 \pi R \sqrt{p^{2}+m^{2}}}\right)\right. \\
-\left(2 \pi R g A_{5}^{(0)}\right)^{2}\left[\frac{e^{-2 \pi R \sqrt{p^{2}+m^{2}}}}{\left(1-e^{-2 \pi R \sqrt{p^{2}+m^{2}}}\right)^{2}}\right] \\
\left.+\left(2 \pi R g A_{5}^{(0)}\right)^{4}\left[\frac{e^{-2 \pi R \sqrt{p^{2}+m^{2}}}}{6\left(1-e^{-2 \pi R \sqrt{p^{2}+m^{2}}}\right)^{2}}+\frac{e^{-4 \pi R \sqrt{p^{2}+m^{2}}}}{\left(1-e^{-2 \pi R \sqrt{p^{2}+m^{2}}}\right)^{4}}\right]\right\} .
\end{gathered}
$$

We see immediately that the vacuum has non-vanishing $\left\langle A_{5}^{(0)}\right\rangle$,

$$
\left\langle A_{5}^{(0)}\right\rangle \sim \frac{1}{R g},
$$

for $m<1 / R$.

Let us now return from considering $U(1)$ gauge group back to $S U(2)$. Nothing much changes as far as the $\Psi$ loop contribution we have just considered $\left(A_{5}^{(0)}\right.$ is just to be replaced by $\left|A_{5}^{(0)}\right| \equiv \sqrt{\operatorname{tr} A_{5}^{2}}$, where the trace is over gauged isospin) but now there are also diagrams involving gauge loops which contribute to the $A_{5}^{(0)}$ effective potential. See Fig. 8. By similar methodology, these give a contribution illustrated in Fig. 9.

We see that there is a competition now between the contribution from gauge loops which prefers a vacuum at $A_{5}^{(0)}=0$ versus the fermion loops which prefer a vacuum at $\left|A_{5}^{(0)}\right| \neq 0$. But clearly if we include sufficiently many identical species of $\Psi$, their contribution must dominate, and $\left|A_{5}^{(0)}\right| \sim$ $1 /(R g)$. Since $A_{5}^{(0)}$ is an isovector, a non-zero expectation necessarily breaks the gauge group $S U(2)$ down to $U(1)$. One can think of this as a caricature 


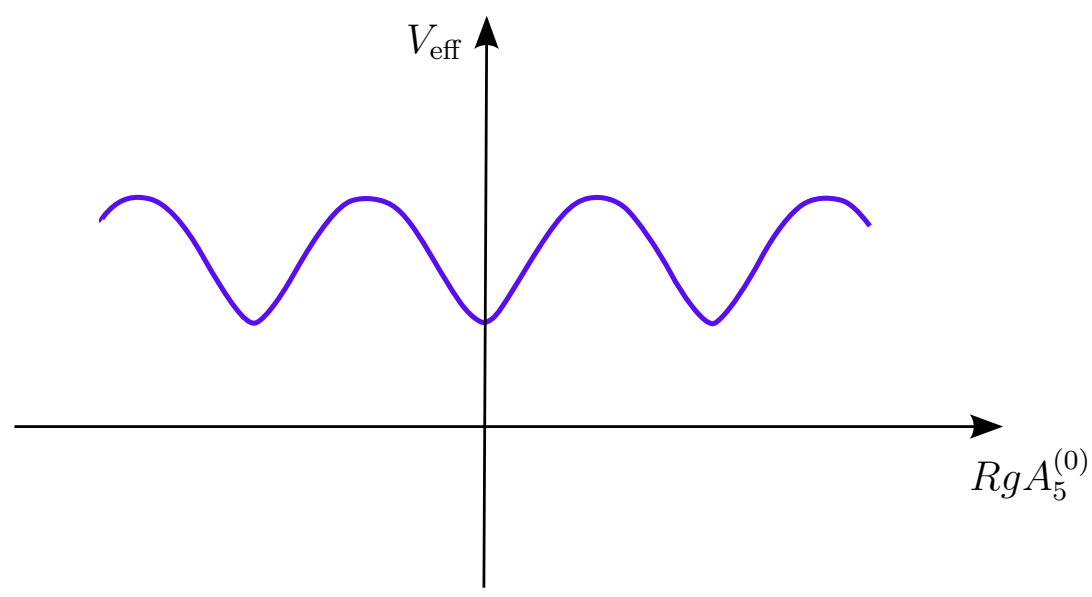

Figure 9: Result of gauge loop contribution to $A_{5}$ potential

of electroweak symmetry breaking where the preserved $U(1)$ is electromagnetism and $A_{5}^{(0)}$ is the Higgs field! We refer to it as "radiative symmetry breaking" (also the "Hosotani mechanism" [12]) because it is a loop effect that sculpted out the symmetry breaking potential.

In this symmetry breaking vacuum or Higgs phase, we can easily estimate the physical mass spectrum,

$$
\begin{aligned}
& m_{\gamma^{(0)}}=0 \\
& m_{W^{ \pm}(0)} \sim \frac{1}{R} \\
& m_{\Psi^{(0)}} \sim \sqrt{m^{2}+\frac{1}{R^{2}}} \underset{m \rightarrow 0}{\longrightarrow} \frac{1}{R} \\
& m_{\mathrm{KK}} \sim \frac{1}{R} \\
& m_{\text {"Higgs" }}^{2} \sim \frac{g^{2}}{32 \pi^{3} R^{3}} .
\end{aligned}
$$

Now this is certainly an interesting story theoretically, but it is surely dangerous to imagine anything like this happening in the real world because we are predicting $m_{\mathrm{KK}} \sim m_{W}$, and such light KK states should already have been seen. However, there is a simple way to make the KK scale significantly 
larger than $m_{W}$, by making

$$
\left\langle A_{5}^{(0)}\right\rangle \ll \frac{1}{R g} .
$$

Note that for small $A_{5}^{(0)}$ we have

$$
\begin{aligned}
& V_{\text {eff }}=V_{\text {eff }}^{\Psi-\text { loop }}+V_{\text {eff }}^{\text {gauge-loop }} \underset{\text { small } a}{\sim} \Lambda R \\
& +\left[c_{1}-c_{2}(m) N\right](R a)^{2}+\left[c_{3}+c_{4}(m) N\right](R a)^{4},
\end{aligned}
$$

where the $c$ 's are order one and positive, and $c_{2}, c_{4}$ depend on the $5 \mathrm{D}$ fermion mass $m$, and $N$ is the number of species of fermions. Now let us tune $m$ to achieve

$$
\begin{aligned}
-c_{1}+c_{2}(m) N & \equiv \varepsilon \ll 1 \\
c_{3}+c_{4}(m) N & \sim \mathcal{O}(1),
\end{aligned}
$$

from which it follows that there is a local minimum of the effective potential (a possibly cosmologically stable, false vacuum) with

$$
A_{5}^{(0)} \sim \frac{\sqrt{\varepsilon}}{g R} .
$$

This yields the hierarchy,

$$
m_{W^{ \pm}} \sim \frac{\sqrt{\varepsilon}}{R} \sim \sqrt{\varepsilon} m_{\mathrm{KK}} .
$$

\section{Orbifolds and Chirality}

If we ask whether our results thusfar could be extended to a realistic model of nature, with the standard model as a low energy limit, we encounter some big problems, not just problems of detail:

a) The previously mentioned chirality problem.

b) Yukawa couplings of the standard model vary greatly. Our low energy fermion modes seem to have Yukawa couplings equal to their gauge coupling, a reasonable cartoon of the top quark but not of other real world fermions.

A very simple way of solving (a) is to replace the fifth dimensional circle by an interval. The two spaces can be technically related by realizing the 


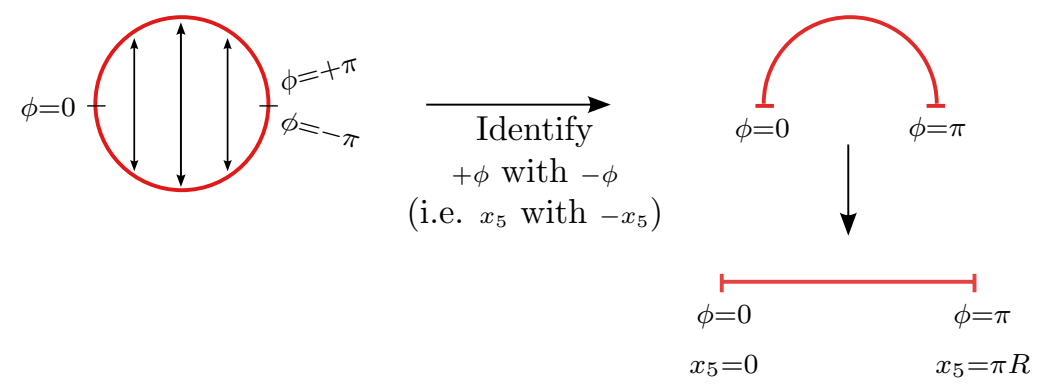

Figure 10: Orbifolding the circle to an interval

interval as an "orbifold" of the circle. This is illustrated in Fig. 10, where the points on the two hemispheres of the circle are identified. Mathematically, we identify the points at $\phi$ or $x_{5}$ with $-\phi$ or $-x_{5}$. In this way the physical interval extends a length $\pi R$, half the circumference of our original circle. This identification is possible if we also assign a "parity" transformation to all the fields, which is respected by the dynamics (i.e. the action). The action we have considered above has such a parity, given by

$$
\begin{array}{lll}
P\left(x_{5}\right)=-x_{5} & P\left(A_{\mu}\right)=+A_{\mu} & P\left(A_{5}^{(0)}\right)=-A_{5}^{(0)} \\
& P\left(\Psi_{\mathrm{L}}\right)=+\Psi_{\mathrm{L}} & P\left(\Psi_{\mathrm{R}}\right)=-\Psi_{\mathrm{R}}
\end{array}
$$

precisely when the $5 \mathrm{D}$ fermion mass vanishes, $m=0$. We consider this case for now.

Ex. Check that the action is invariant under this parity transformation.

With such a parity transformation we continue to pretend to live on a circle, but with all fields satisfying

$$
\Phi\left(x^{\mu},-x_{5}\right)=P(\Phi)\left(x^{\mu}, x_{5}\right) .
$$

That is, the degrees of freedom for $x_{5}<0$ are merely a reflection of degrees of freedom for $x_{5}>0$, they have no independent existence. Of course we also require circular periodicity,

$$
\Phi\left(x^{\mu}, \phi+2 \pi\right)=\Phi\left(x^{\mu}, \phi\right) .
$$

These conditions specify "orbifold boundary conditions" on the interval, derived from the the circle, which of course has no boundary. 
We can write out the mode decompositions (in almost axial gauge) for all the fields subject to orbifold boundary conditions,

$$
\begin{array}{lll}
A_{\mu}(x, \phi) & =\sum_{n=0}^{\infty} A_{\mu}^{(n)}(x) \cos (n \phi) & \\
A_{5}(x, \phi) & =0 & \text { Lost "Higgs"! } \\
\Psi_{\mathrm{L}}(x, \phi) & =\sum_{n=0}^{\infty} \Psi_{\mathrm{L}}^{(n)}(x) \cos (n \phi) & \\
\Psi_{\mathrm{R}}(x, \phi) & =\sum_{n=1}^{\infty} \Psi_{\mathrm{R}}^{(n)}(x) \sin (n \phi) & \text { Lost } \Psi_{\mathrm{R}}^{(0)} !
\end{array}
$$

One unfortunate consequence we see is that $A_{5}$ has no modes, in particular orbifolding has eliminated our candidate Higgs! The good consequence is for the chirality problem, in that the massless right-handed fermion is eliminated, only the massless left handed fermion mode is left. The low energy effective theory below $1 / R$ is just

$$
S_{\text {eff }} \underset{E \ll \frac{1}{R}}{=} 2 \pi R \int d^{4} x\left\{-\frac{1}{4}\left(F_{\mu \nu}^{(0)}\right)^{2}+\bar{\Psi}_{\mathrm{L}}^{(0)} i D_{\mu} \gamma^{\mu} \Psi_{\mathrm{L}}^{(0)}\right\} .
$$

With $S U(2)$ gauge group, if $\Psi$ is an isodoublet (so that $\Psi_{L}^{(0)}$ is an isodoublet), the only possible gauge invariant mass term for the light mode,

$$
\Psi_{\mathrm{L}_{\alpha}}^{i} \Psi_{\mathrm{L}}^{j} \epsilon_{i j} \epsilon^{\alpha \beta}
$$

vanishes by fermi statistics. Therefore we apparently have a chiral effective gauge theory below $1 / R$. Unfortunately this theory is afflicted by a subtle non-perturbative "Witten anomaly", so the theory is really unphysical. However, if we consider $\Psi$ to be in the isospin $3 / 2$ representation, we again get a chiral gauge theory, but now not anomalous in any way.

Having seen that the chirality problem is soluble, we need to recover our Higgs field. (For discussion of related mechanisms and further references see the TASI review of Ref. [13.) To do this we must enlarge our starting gauge group, from

$$
S U(2) \cong S O(3)
$$


to $S O(4)$. Gauge fields are conveniently thought of as anti-symmetric matrices, $A_{M}^{i j}$, in the fundmental gauge indices $i, j=1,2,3,4$. For simplicity we choose fermions in the fundamental representation, $\Psi^{i}$. The action,

$$
\begin{aligned}
S=\operatorname{tr} \int d^{4} x \int d x_{5} & \left\{-\frac{1}{4} F_{\mu \nu} F^{\mu \nu}+\frac{1}{2}\left(\partial_{5} A_{\mu}\right)^{2}+\frac{1}{2}\left(D_{\mu} A_{5}^{(0)}\right)^{2}\right. \\
+ & \left.\bar{\Psi} i D_{\mu} \gamma^{\mu} \Psi-\bar{\Psi} \gamma_{5} \partial_{5} \Psi+i g \bar{\Psi}_{i} A_{5}^{i j(0)} \gamma_{5} \Psi_{j}\right\},
\end{aligned}
$$

is invariant under the orbifold parity given by

$$
\begin{array}{llll}
P\left(A_{\mu}^{\hat{i} \hat{j}}\right)=+A_{\mu}^{\hat{i} \hat{j}} & P\left(A_{5}^{\hat{i} \hat{j}}\right)=-A_{5}^{\hat{i} \hat{j}} & P\left(\Psi_{\mathrm{L}}^{\hat{i}}\right)=+\Psi_{\mathrm{L}}^{\hat{i}} & P\left(\Psi_{\mathrm{R}}^{\hat{i}}\right)=-\Psi_{\mathrm{R}}^{\hat{i}} \\
P\left(A_{\mu}^{\hat{i} 4}\right)=-A_{\mu}^{\hat{i} 4} & P\left(A_{5}^{\hat{i} 4}\right)=+A_{5}^{\hat{i} 4} & P\left(\Psi_{\mathrm{L}}^{4}\right)=-\Psi_{\mathrm{L}}^{4} & P\left(\Psi_{\mathrm{R}}^{4}\right)=+\Psi_{\mathrm{R}}^{4},
\end{array}
$$

where $\hat{i}, \hat{j}=1,2,3$.

Ex. Check by mode decomposition that this leaves 4D massless fields,

$$
A_{\mu}^{\hat{i} \hat{j}(0)}, \quad A_{5}^{\hat{i} 4(0)}, \quad \Psi_{\mathrm{L}}^{\hat{i}(0)}, \quad \Psi_{\mathrm{R}}^{4(0)},
$$

that is, a $4 \mathrm{D} S O(3)$ gauge field, a $4 \mathrm{D}$ Higgs triplet of $S O(3)$, a left-handed fermion triplet of $S O(3)$, and a right-handed singlet of $S O(3)$.

This illustrates how (orbifold) boundary conditions on extra dimensions can break the gauge group of the bulk of the extra dimensions. The lowenergy effective theory is given by

$$
\begin{aligned}
S_{\text {eff }}=2 \pi R \int & d^{4} x\left\{-\frac{1}{4} F_{\mu \nu}^{(0)} F^{\mu \nu(0)}+\frac{1}{2}\left(D_{\mu} A_{5}^{\hat{i} 4(0)}\right)^{2}\right. \\
+ & \bar{\Psi}_{\mathrm{L}}^{\hat{i}(0)}\left(i D_{\mu} \gamma^{\mu} \Psi_{\mathrm{L}}^{(0)}\right)_{\hat{i}}+\bar{\Psi}_{\mathrm{R}}^{4(0)} i \partial_{\mu} \gamma^{\mu} \Psi_{\mathrm{R}}^{4(0)} \\
+ & \left.i g\left(\bar{\Psi}_{\mathrm{L}_{\hat{i}}^{(0)}}^{(\hat{i}} A_{5}^{\hat{i} 4(0)} \Psi_{\mathrm{R} 4}^{(0)}+\bar{\Psi}_{\mathrm{R} 4}^{(0)} A_{5}^{\hat{i} 4(0)} \Psi_{\mathrm{L}_{\hat{i}}}^{(0)}\right)\right\}
\end{aligned}
$$

This contains $4 \mathrm{D} S O(3)$ gauge theory with two different representations of Weyl fermions Yukawa-coupled to a Higgs field. This again bears some resemblence to the standard model if we think of the fermion as the left and right handed "top" quark. But what of the second problem we identified, (b), that the standard model contains some fermions with much smaller Yukawa couplings than gauge coupling? Such fermions can arise by realizing them very differently in the higher-dimensional set-up. The simplest example is illustrated in Fig. 11, where beyond the fields we have thusfar considered, 


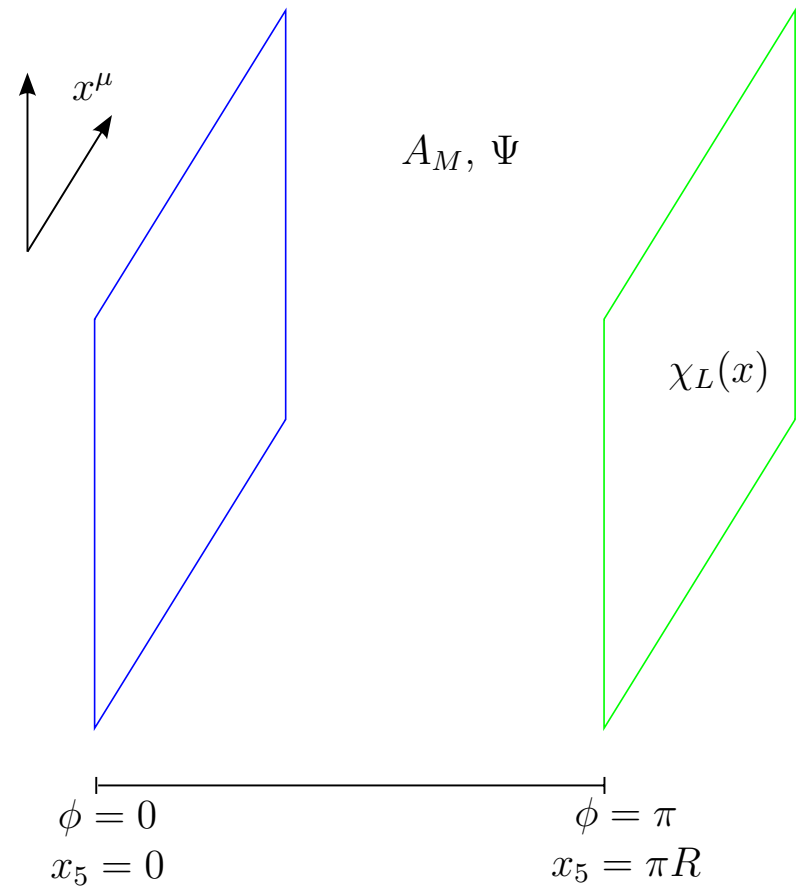

Figure 11: Orbifolded higher dimensional spacetime (with boundaries)

which live in the "bulk" of the 5D spacetime, there is a 4D Weyl fermion precisely confined to one of the 4D boundaries of the 5D spacetime, say $\phi=\pi$. It can couple to the gauge field evaluated at the boundary if it carries some non-trivial representation, say triplet. This represents a second way in which the chirality problem can be solved, localization to a physical 4D subspace or "3-brane" (a " $p$ "-brane has $p$ spatial dimensions plus time), in this case the boundary of our 5D spacetime. The new fermion has action,

$$
S_{\chi}=\int d^{4} x \bar{\chi}_{\mathrm{L}}^{\hat{i}}(x)\left[i \partial_{\mu} \delta^{\hat{i} \hat{j}}+g A_{\mu}^{\hat{i} \hat{j}}(x, \phi=\pi)\right] \chi_{\mathrm{L}}^{\hat{j}}(x) .
$$

At low energies, $E \ll 1 / R$, this fermion will have identical gauge coupling as the $\Psi^{(0)}$ triplet, but it will have no Yukawa coupling, thereby giving a crude representation of a light fermion of the standard model.

Well, there are other tricks that one can add to get closer and closer to the real world. Ref. [14 gives a nice account of many model-building issues and further references. I want to move in a new direction. 


\section{Matching 5D to 4D couplings}

Let us study how effective 4D couplings at low energies emerge from the starting 5D couplings. Returning to pure $S U(2)$ Yang-Mills on an extradimensional circle, we get a low-energy 4D theory,

$$
S_{4 \mathrm{eff}} \underset{E \ll \frac{1}{R}}{\sim} 2 \pi R \int d^{4} x\left\{-\frac{1}{4} F_{\mu \nu}^{(0)} F^{\mu \nu(0)}+\frac{1}{2}\left(D_{\mu} A_{5}^{(0)}\right)^{2}\right\} .
$$

The fields are clearly not canonically normalized, even though the 5D theory we started with was canonically normalized. We can wavefunction renormalize the $4 \mathrm{D}$ effective fields to canonical form,

$$
\varphi \equiv A_{5}^{(0)} \sqrt{2 \pi R}, \quad \bar{A}_{\mu} \equiv A_{\mu}^{(0)} \sqrt{2 \pi R},
$$

and see what has happened to the couplings,

$$
\begin{gathered}
S_{4 \mathrm{eff}}=2 \pi R \int d^{4} x\left\{-\frac{1}{4}\left(\partial_{\mu} A_{\nu}^{a(0)}-\partial_{\nu} A_{\mu}^{a(0)}-i g_{5} \epsilon^{a b c} A_{\mu}^{b(0)} A_{\nu}^{c(0)}\right)^{2}\right. \\
\left.+\frac{1}{2}\left(\partial_{\mu} A_{5}^{(0)}-i g_{5} A_{\mu}^{(0)} A_{5}^{(0)}\right)^{2}\right\} \\
=\int d^{4} x\left\{-\frac{1}{4}\left(\partial_{\mu} \bar{A}_{\nu}^{a}-\partial_{\nu} \bar{A}_{\mu}^{a}-i \frac{g_{5}}{\sqrt{2 \pi R}} \epsilon^{a b c} \bar{A}_{\mu}^{b} \bar{A}_{\nu}^{c}\right)^{2}\right. \\
\left.+\frac{1}{2}\left(\partial_{\mu} \varphi-i \frac{g_{5}}{\sqrt{2 \pi R}} \bar{A}_{\mu} \varphi\right)^{2}\right\} .
\end{gathered}
$$

From this we read off the effective 4D gauge coupling,

$$
g_{4 \mathrm{eff}}=\frac{g_{5}}{\sqrt{2 \pi R}} .
$$

Ex. Check that this is dimensionally correct, that $4 \mathrm{D}$ gauge couplings are dimensionless while 5D gauge couplings having units of $1 / \sqrt{\text { mass. }}$.

For experimentally measured gauge couplings, roughly order one, we require

$$
g_{5} \sim \mathcal{O}(\sqrt{2 \pi R})
$$

\section{$7 \quad 5 \mathrm{D}$ Non-renormalizability}

Now, having couplings with negative mass dimension is the classic sign of non-renormalizability, and as you can easily check it happens rather readily 
in higher dimensional quantum field theory. There are various beliefs about non-renormalizable theories:

a) A non-renormalizable quantum field theory is an unmitigated disaster. Throw the theory away at once. Only a few people still hold to this incorrect viewpoint.

b) A non-renormalizable quantum field theory can only be used classically, for example in General Relativity where $G_{\text {Newton }}$ has negative mass dimension. All quantum corrections give nonsense. This incorrect view is held by a surprisingly large number of people.

c) The truth (what I believe): Non-renormalizable theories with couplings, $\kappa$, with negative mass dimension can make sense as effective field theories, working pertubatively in powers of the dimensionless small parameter, $\kappa(\text { Energy })^{\mathrm{n}}$, where $-n$ is the mass dimension of $\kappa$. To any fixed order in this expansion, one in fact has all the advantages of renormalizable quantum field theory. There are even meaningful finite quantum computations one can perform. In fact we have just done one in computing the $A_{5}$ quantum effective potential. But of course there is a price: the whole procedure breaks down once the formal small parameter is no longer small, $E \sim 1 / \kappa^{1 / n}$. At higher energies the effective field theory is useless and must be replaced by a more fundamental and better behaved description of the dynamics.

Ex. Learn (non-renormalizable) effective field theory at the systematic technical level as well as a way of thinking. A good place to start is the chiral Lagrangian discussion of soft pions in Ref. [15].

In more detail, perturbative expansions in effective field theory will have expansion parameters, $\kappa(\text { Energy })^{\mathrm{n}}$, divided by extra numerical factors such as 2's or $\pi$ 's. These factors are parametrically order one, but enough of them can be quantitatively significant. These factors can be estimated from considerations of phase space. I will just put these factors in correctly without explanation.

Ex. Learn the art of naive dimensional analysis, including how to estimate the 2's and $\pi$ 's (for some discussion in the extra-dimensional context see Ref. [16]). Use this in your work on extra dimensions.

Our findings so far are summarized in Fig. 12. The non-renormalizable effective field theory of 5D gauge theory breaks down when the formal small parameter, $E g_{5}^{2}$, gets large, that is we can define a maximum cutoff on its validity, $\Lambda_{\mathrm{UV}}$, max $\sim 16 \pi^{2} / g_{5}^{2}$. The $5 \mathrm{D}$ effective theory cannot hold above this scale and must be replaced by a more fundamental theory. Let us say this happens at $\Lambda_{\mathrm{UV}} \leq \Lambda_{\mathrm{UV}}$, max. From here down to $1 / R$ we have $5 \mathrm{D}$ effective 


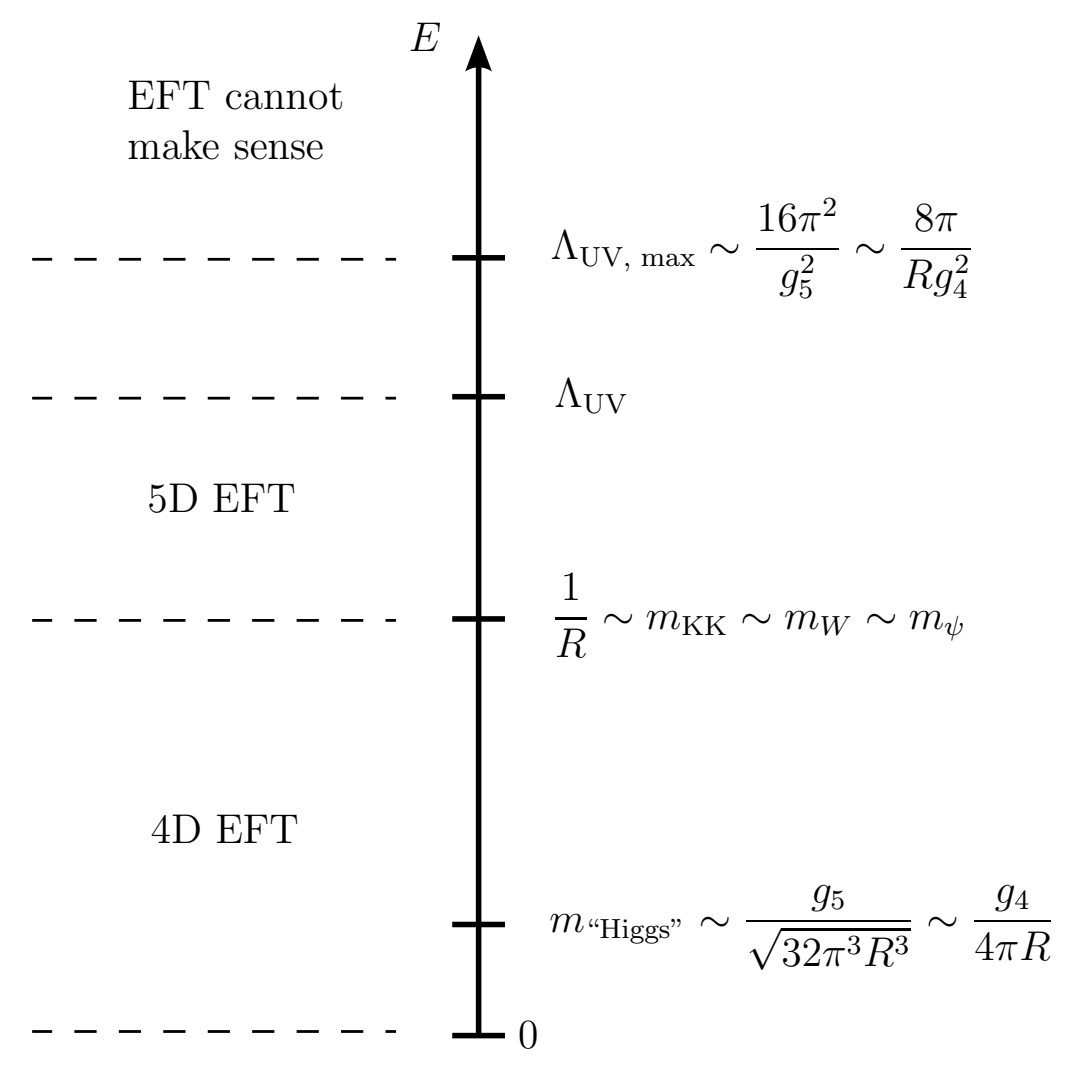

Figure 12: Natural scales of the 5D gauge theory

field theory, and below $1 / R$ we have $4 \mathrm{D}$ effective field theory.

We found it interesting that a Higgs-like candidate emerged from 5D gauge fields because it suggested a way of keeping the 4D scalar naturally light, namely by identifying it as part of a higher-dimensional vector field. But given that $\Lambda_{\mathrm{UV}} \leq \Lambda_{\mathrm{UV}}$, max $\sim \mathcal{O}\left(8 \pi /\left(R g_{4}^{2}\right)\right)$, and 4D gauge couplings are measured to be not much smaller than one, we must ask how well this extradimensional picture is doing at addressing the naturalness problem of the Higgs. In Fig. 13 we make the comparison with purely 4D field theory with a UV cutoff imposed. We see that in the purely 4D scenario one naturally predicts a weak scale $v \sim \mathcal{O}\left(\Lambda_{\mathrm{UV}} / 4 \pi\right)$, while with the $5 \mathrm{D}$ regime included, $v \sim \mathcal{O}\left(g_{4} \Lambda_{\mathrm{UV}} / 8 \pi\right)$, which is parametrically better. Numerically, it does not look like much improvement, and indeed one can do better, but we do not pursue that here. 


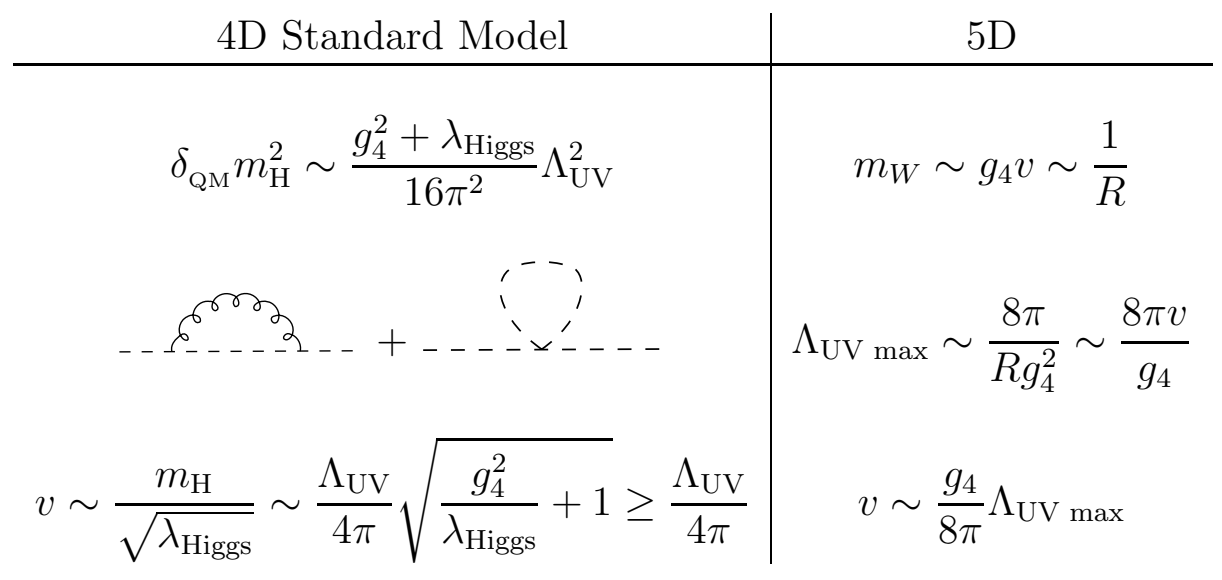

Figure 13: Natural relationship between $W$ mass and cutoff

\section{Gravity-Gauge Unification and the Radion}

The non-renormalizable effective field theory of quantum General Relativity, whose small expansion parameter is $E^{2} G_{\text {Newton }}$, is expected to be "UVcompleted" by string theory, just as the Fermi theory of weak interactions is completed by the renormalizable electroweak theory. One price of calculable string theories is that they predict the existence of extra dimensions! You do not have to ask for extra dimensions, they are forced on you as the price of maintaining the stringy gauge symmetries needed to tame the higher spins in the theory. In fact the extra dimensions are required in order to cancel quantum anomalies in the stringy gauge symmetries [5]. However, string theory has been notoriously unpredictive about the size and shape of the extra dimensions, so we must gedanken experiment, or really experiment, with the possibilities. Since string theory contains a regime of (higher-dimensional) General Relativity, the size and shape of the extra dimensions is dynamical as is all of spacetime. This raises several interesting issues, which we study below.

Let us begin by returning to 5D spacetime with circular extra dimension. The 5D gravitational field is given by the metric function on this spacetime, in 
terms of which we can write infinitesimal distances in arbitrary coordinates,

$$
\begin{aligned}
d s^{2} & =G_{M N}(X) d X^{M} d X^{N} \\
& =G_{M N}\left(X\left(X^{\prime}\right)\right) \frac{\partial X^{M}}{\partial X^{\prime M^{\prime}}} d X^{\prime M^{\prime}} \frac{\partial X^{N}}{\partial X^{N^{\prime}}} d X^{N^{\prime}} \\
& =G_{M^{\prime} N^{\prime}}\left(X^{\prime}\right) d X^{M^{\prime}} d X^{N^{\prime}}
\end{aligned}
$$

From this, we deduce how the metric field transforms under 5D general coordinate transformations (the gauge symmetry of 5D General Relativity) in order to maintain the invariance of physical distances,

$$
G_{M^{\prime} N^{\prime}}\left(X^{\prime}\right)=G_{M N}\left(X\left(X^{\prime}\right)\right) \frac{\partial X^{M}}{\partial X^{\prime M^{\prime}}} \frac{\partial X^{N}}{\partial X^{\prime N^{\prime}}} .
$$

Expanding around flat (cylindrical) spacetime,

$$
G_{M N}(X)=\eta_{M N}+h_{M N}(X)
$$

and working to leading order in $h_{M N}$ and small coordinate transformations,

$$
X^{M}=X^{M}-\varepsilon^{M}(X)
$$

we see that the general coordinate transformation of the metric reduces to

$$
h_{M N}^{\prime}(X)=h_{M N}(X)+\partial_{M} \varepsilon_{N}+\partial_{N} \varepsilon_{M} .
$$

Again, we can go to an almost axial gauge of the form,

$$
\begin{aligned}
& h_{\mu 5}(x, \phi)=h_{\mu 5}^{(0)}(x) \\
& h_{55}(x, \phi)=h_{55}^{(0)}(x) \\
& h_{\mu \nu}(x, \phi)=h_{\mu \nu}^{(0)}(x)+\sum_{n=1}^{\infty}\left(h_{\mu \nu}^{(n)}(x) e^{i n \phi}+\text { c.c. }\right),
\end{aligned}
$$

where we use coordinates

$$
X=\left(x^{\mu}, \phi\right) .
$$

Ex. Show how this is to be done using the linearized transformation of the metric. 


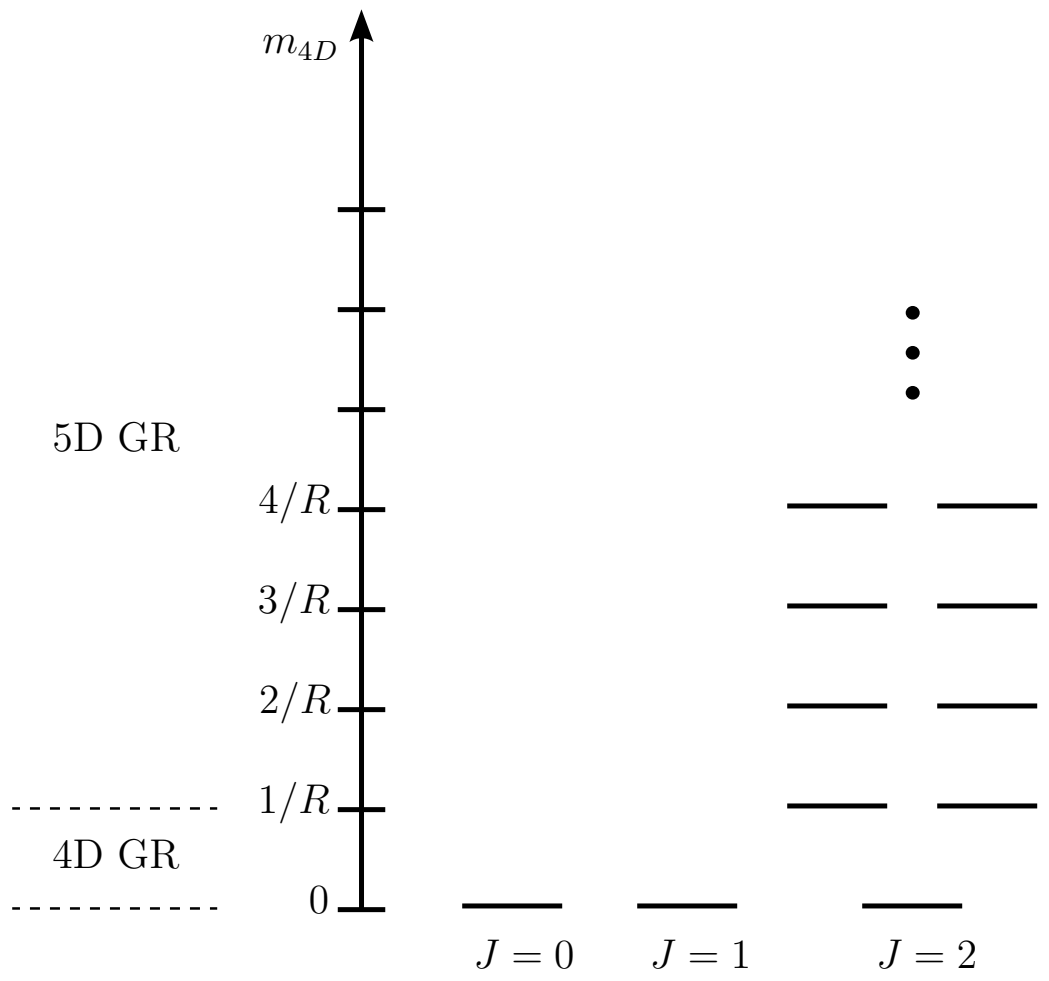

Figure 14: KK 4D spectrum of 5D metric decomposition

Since the 5D Einstein action,

$$
S_{5 D \text { Einstein }}=\int d^{5} X \frac{\sqrt{G} \mathcal{R}}{G_{N}^{5 D}},
$$

written in terms of the 5D Ricci scalar curvature $\mathcal{R}$ contains only terms with derivatives, the $h_{M N}^{(0)}(x)$ must be massless $4 \mathrm{D}$ fields, without any potential at all. The $h_{M N}^{(n)}$ have $n / R$ masses as usual. These facts are illustrated in Fig. 14. Of course once one goes beyond the linearized approximation, all these fields are interacting. The interacting massless vector field, $h_{\mu 5}^{(0)}(x)$, must therefore have a protective gauge symmetry.

Ex. Check that in almost axial gauge there is a residual unfixed symmetry,

$$
h_{\mu 5}^{\prime}(X)=h_{\mu 5}(X)+\partial_{\mu} \varepsilon_{5}(x) .
$$

Because of this gauge invariance, inherited from 5D general coordinate in- 
variance, the quadratic terms involving $h_{\mu 5}^{(0)}(x)$ in the low energy $4 \mathrm{D}$ effective action must be just the Maxwell action. This is part of the original KaluzaKlein idea, 4D gauge fields and 4D General Relativity (protected by the residual 4D general coordinate invariance) both emerge from a unifed 5D action upon compactification. The 4D effective action is tightly constrained by the protective symmetries to be of Maxwell-Einstein type, as can be checked by plugging in the massless modes as usual. It is easiest to just check this at quadratic order in the action and then use the residual symmetries. Since the vector field gauge symmetry corresponds to shifts in the extra dimensional circle (and because there is precisely one emergent vector field), the emergent gauge group is $U(1)$. Charged matter must correspond to states which transform under such extra-dimensional translations. These are precisely the KK excitations which carry non-zero extra-dimensional momentum.

It is intriguing to see how "internal" gauge groups can emerge from the symmetries of the extra-dimensional geometry. One might wonder whether more complex and realistic gauge structure, as well as chiral 4D massless charged matter, can emerge from extra-dimensional geometry. While higher dimensional set-ups go in this direction, it is in general a very difficult game. However, in one way of analysing heterotic string theory realistic gauge structure does emerge from several KK $U(1)$ gauge fields with special stringy enhancements to non-abelian form [6]. In this sense the old Kaluza-Klein program of unification of gauge theory (just electromagnetism in the early days) and gravity is alive and well.

There is one more 4D massless state which does not follow from a residual symmetry, and to that extent is unexpected at first sight, namely $h_{55}^{(0)}(x)$. To repeat, it has no potential because the $5 \mathrm{D}$ action has only derivative terms, and this mode only has $x$-derivatives, being independent of the extra dimension by gauge-fixing. This 4D scalar can therefore have any VEV,

$$
\left\langle h_{55}^{(0)}\right\rangle=\xi .
$$

To see what this VEV means physically, note that for the simple VEVs,

$$
\left\langle h_{\mu \nu}^{(0)}\right\rangle=0, \quad\left\langle h_{\mu 5}^{(0)}\right\rangle=0,
$$

the $\mathrm{VEV}$ of the 5D geometry is given by

$$
\begin{aligned}
\left\langle d s^{2}\right\rangle & =\eta_{\mu \nu} d x^{\mu} d x^{\nu}-(1+\xi) d x_{5} d x_{5} \\
& =\eta_{\mu \nu} d x^{\mu} d x^{\nu}-(1+\xi) R^{2} d \phi^{2}
\end{aligned}
$$




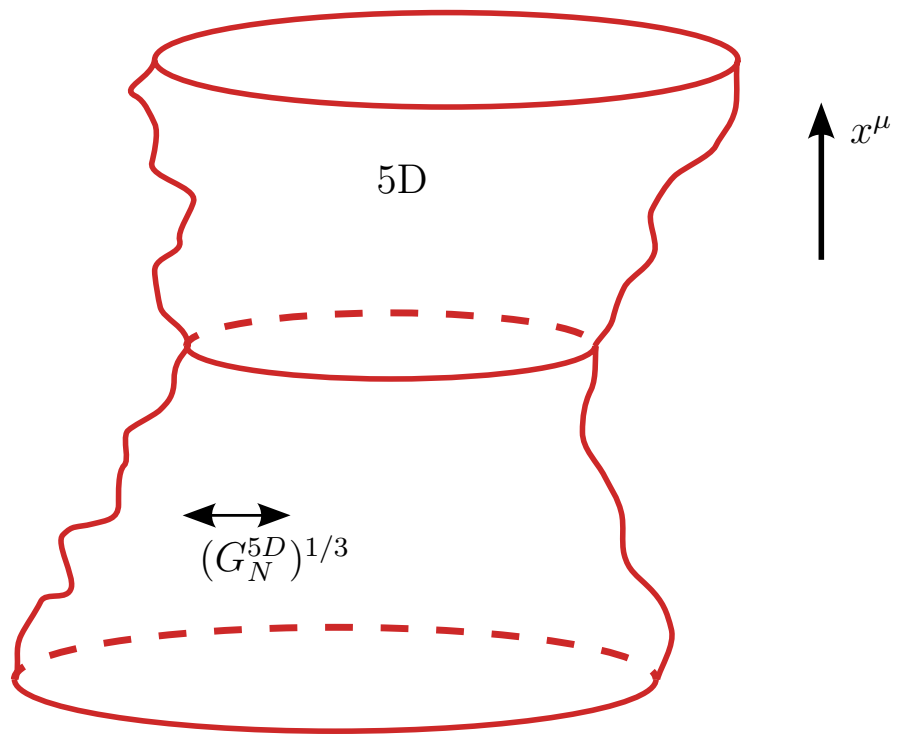

Figure 15: Fluctuations of dynamical radius (radion)

which is just the geometry of an undeformed (flat) cylinder with physical radius $\sqrt{1+\xi} R$. Thus, fluctuations in the scalar field correspond to a dynamical radius for the extra dimension, as illustrated in Fig. 15. The quantum of this scalar is therefore referred to as the "radion".

We are used to scalar fields without potentials in the form of Goldstone bosons. But in these cases, any VEV in field space is equivalent to any other by the associated symmetry. The radion field space is an example of a "moduli space" of physically inequivalent vacua (differing radii are physically quite different obviously). The radion is an example of a "modulus". The string theories that have been studied start life with very large moduli spaces of inequivalent vacua. Thus even a unique theory can lose predictivity in the maze of physically inequivalent vacua Nature has to choose from.

The good news is that we see a cartoon of the 4D ingredients needed for the real world emerging from our very simple unified 5D example: 4D scalars, $4 \mathrm{D}$ gauge theory and $4 \mathrm{D}$ gravity. If one throws in supersymmetry, another key ingredient of superstring theory, one must necessarily have fermions as well. The size and shape of the extra dimensions, and the low energy $4 \mathrm{D}$ world they produce, is determined by the moduli VEVs. Small corrections to the vanishing potential of moduli space can actually favor one or a discrete set 
of vacua, yielding greater predictivity (in string theory right now "greater" does not mean great). Let us continue with our 5D cylinder to see a simple example of such small corrections, in this case from quantum effects related to the famous Casimir effect.

\section{Modulus Stabilization}

Let us add a 5D fermion to the (gravitating) cylinder. Our old effective potential calculation from fermion loops, now dropping $A_{5}$ since there are no $5 \mathrm{D}$ gauge fields, is given by

$$
V_{\mathrm{eff}} \sim \Lambda R-4 \int \frac{d^{4} p}{(2 \pi)^{4}} \ln \left(1-e^{-2 \pi R \sqrt{p^{2}+m^{2}}}\right) .
$$

In the 5D general relativistic context we should consider the 1-fermion-loop induced effective potential for the dynamical radius VEV, $R$. Recall, $\Lambda$ contains all UV divergences in this calculation. The renormalized value of $\Lambda$ is frequently tuned to zero (more on this later) so that crudely,

$$
V_{\text {eff }} \underset{m \gg \frac{1}{R}}{\sim} e^{-2 \pi R m} \int_{0}^{m} d^{4} p \sim m^{4} e^{-2 \pi R m} .
$$

Now, let us assume that there is also a 5D scalar boson as well and add its quantum-loop contribution to the $R$ effective potential. In complete analogy to our fermion loop computation, except for a sign due to the different statistics,

$$
V_{\text {eff }} \sim m^{4} e^{-2 \pi R m}-\mu^{4} e^{-2 \pi R \mu} .
$$

Upon minimizing the effective potential with respect to the dynamical radius,

$$
e^{2 \pi R(m-\mu)} \sim\left(\frac{m}{\mu}\right)^{5}, \quad R \sim \frac{5 \ln \left(\frac{m}{\mu}\right)}{2 \pi(m-\mu)} .
$$

That is the corrections have selected a particular radius from the original moduli space!

The general conclusion is that in (non-supersymmetric) extra dimensions small corrections can generate an effective potential for moduli which stabilizes the size and shape of the extra dimensions. This radius can readily 
be moderately larger than the fundamental length scale of the cutoff of nonrenormalizable higher-dimensional effective field theory. In the present example, this is achieved by taking the matter masses, $m, \mu$, to be moderately lighter than the mass scale of the cutoff.

If the present example is orbifolded so that the extra dimension is an interval, one can also add a constant "potential" to be localized to one of the boundaries. Since such a localized term is insensitive to $R$, it will just contribute an $R$-independent constant to the effective potential. Such a constant seems irrelevant from the point of view of the radion, but it is important when the effective dynamics of 4D General Relativity is also taken into account, as we discuss in the next section.

\section{The Cosmological Constant (Problem)}

The dominant interactions in 4D General Relativity at long distances include not just the Einstein action but also a cosmological constant term,

$$
S=\int d^{4} x \sqrt{-g}\left\{-\lambda+\frac{\mathcal{R}}{16 \pi G_{N}}\right\} .
$$

In flat space quantum matter generates zero-point energies or vacuum energy, corrected by interactions in all possible ways, but we usually throw this away as a physically irrelevant field-independent constant in the effective action. But when coupled to gravity, all vacuum energy from matter and radiation contributes to the cosmological term. If we trust the standard model up to $\mathrm{TeV}$ energies, one naturally expects contributions to $\lambda$ of order at least $\mathrm{TeV}^{4}$. Crudely, in the presence of such a term Einstein's Equations read

$$
\mathcal{R} \sim G_{N} \lambda
$$

where the left-hand side is a measure of the curvature of spacetime. In particular, flat Minkowski spacetime is not a solution to Einstein's equations for $\lambda \neq 0$. If $\lambda \sim \mathrm{TeV}^{4}$, the radius of curvature of spacetime would be

$$
\frac{1}{\sqrt{\mathcal{R}}} \sim \frac{M_{P l}}{\sqrt{\lambda}} \sim \frac{M_{P l}}{\mathrm{TeV}^{2}} \sim 1 \mathrm{~mm} !
$$

This would be tremendously at odds with even everyday experience, where space appears to satisfy Euclid's postulates to excellent approximation. 
We therefore have to assume all the varied contributions to the cosmological term cancel to very high precision for some mysterious reason. We will assume this from now on to make contact with the observed universe. This unnatural feature is called the Cosmological Constant Problem [17].

In the modulus stabilization example of the last section, the value of $V_{\text {eff }}$ at its minimum represents (in general, a contribution to) the infrared $4 \mathrm{D}$ cosmological constant. As pointed out at the end of that section, in the orbifolded version one can also add an $R$-independent constant to $V_{\text {eff }}$, originating from a boundary-localized constant potential. By fine-tuning the value of this constant we can ensure that the minimal $V_{\text {eff }}$ vanishes, that is, the $4 \mathrm{D}$ effective cosmological constant is zero (or very small). The ugliness of having to do this tuning reflects the unresolved cosmological constant problem.

Just like 4D General Relativity, 5D General Relativity can also have a cosmological term,

$$
S=\int d^{5} X \sqrt{G}\left\{\frac{\mathcal{R}}{G_{N}^{5 D}}-\Lambda\right\} .
$$

Now what we have learned in gauge theory is generally true: $4 \mathrm{D}$ effective couplings derive from $5 \mathrm{D}$ couplings, but they are not the same thing. In particular, our tuning of the $4 \mathrm{D}$ cosmological term, $\lambda$, to be very nearly zero does not indicate that $\Lambda$ is near zero (in fact we will see this explicitly below). The most natural thing is to therefore assume that $\Lambda \neq 0$ and see what happens.

In our $5 \mathrm{D}$ cylinder example, let us treat $\Lambda$ as a small perturbation, where at zeroth order we have a cylindrical solution to Einstein's equations,

$$
\left\langle d s^{2}\right\rangle=\eta_{\mu \nu} d x^{\mu} d x^{\nu}-R_{\text {phys }}^{2} d \phi^{2} .
$$

Therefore the cosmological term in the 5D action becomes

$$
S \ni \int d^{4} x \int_{0}^{2 \pi} d \phi R(-\Lambda)=\int d^{4} x(-2 \pi R \Lambda),
$$

which looks precisely like one of our old contributions to the radion $(R)$ effective potential. In fact we saw that there were divergent renormalizations of this $\Lambda$ from $5 \mathrm{D}$ matter loops, again suggesting that the renormalized $\Lambda$ should naturally be significant. 
Once we decide that $\Lambda$ is not very small, we really should consider its effects in determining the 5D geometry. It cannot be treated perturbatively, we must resolve Einstein's equations in the presence of the 5D cosmological constant. The same is true for any boundary-localized potential terms coupled to 5D gravity.

\section{Warped Compactification}

We will study the simplest model where we include the higher dimensional cosmological term, known as the Randall-Sundrum I (RS1) model [18] [19. It is also a good prototype of more complex constructions.

Rather than continue with the cylindrical spacetime we return to the orbifolded variant. The 5D gravitational action of the "bulk" of the spacetime is still given by

$$
S_{\text {bulk }}=\int d^{4} x \int_{-\pi}^{+\pi} d \phi \sqrt{G}\left\{\frac{\mathcal{R}}{G_{N}^{5 D}}-\Lambda\right\} .
$$

We can assign the orbifold parities as +1 for $G_{\mu \nu}$ and $G_{55}$ and -1 for $G_{\mu 5}$, which is respected by the action. In almost axial gauge then, $G_{\mu 5}^{(0)}(x)=0$ by the parity. Therefore in the orbifolded set-up we automatically have no KK massless gauge boson emerging in the $4 \mathrm{D}$ effective theory. Of course there can be even more dimensions in non-minimal set-ups from which such gauge bosons might emerge. Here, when we need gauge bosons we will just add them at the $5 \mathrm{D}$ level. The radion does remain after orbifolding since $G_{55}$ is parity even.

The orbifolded set-up also has "branes" or boundaries of 5D spacetime, namely the $4 \mathrm{D}$ subspaces at $\phi=0, \pi$. In general there can be $4 \mathrm{D}$ actions localized to these branes for the 5D fields. In fact such actions must be there since they are renormalized by 5D loops 20. The leading terms in these brane actions are "tensions", which look like localized cosmological terms,

$$
S_{\text {brane }(i)}=-\int d^{4} x \sqrt{-g^{(i)}} T^{(i)},
$$

where

$$
\begin{aligned}
& g_{\mu \nu}^{(1)}(x)=G_{\mu \nu}(x, \phi=0) \\
& g_{\mu \nu}^{(2)}(x)=G_{\mu \nu}(x, \phi=\pi),
\end{aligned}
$$


and the $T^{(i)}$ are constant tensions. The induced 4D metrics define distances along the branes, for example,

$$
d s_{(1)}^{2}=G_{\mu \nu}(x, \phi=0) d x^{\mu} d x^{\nu},
$$

since $d \phi=0$ along the brane.

Since we are looking for solutions to Einstein's equations that might fit the vacuum of the real world, let us try the ansatz that the $5 \mathrm{D}$ metric should respect at least 4D Poincare invariance,

$$
d s^{2}=e^{-2 \sigma(\phi)} \eta_{\mu \nu} d x^{\mu} d x^{\nu}-R^{2} d \phi^{2} .
$$

Here, $\eta_{\mu \nu}$ is the $4 \mathrm{D}$ Minkowski metric, and we have chosen the extra-dimensional coordinate to be proportional to proper distance. The prefactor to $\eta_{\mu \nu}$ is written as an exponential as a convenient convention and is called the "warp factor". Its potential $\phi$-dependence means that the higher-dimensional geometry cannot be defined as a product geometry of 4D Minkowski space and some purely extra-dimensional geometry, but rather all the dimensions are entangled. Plugging this ansatz into the equations of motion following from our bulk plus brane actions, one finds

$$
\begin{aligned}
6 \sigma^{\prime 2} & =-\frac{\Lambda}{4 M_{5}^{3}} \equiv 6 k^{2} \\
3 \sigma^{\prime \prime} & =\frac{T^{(1)}}{4 M_{5}^{3}} \delta(R \phi)+\frac{T^{(2)}}{4 M_{5}^{3}} \delta(R(\phi-\pi)),
\end{aligned}
$$

where we define a 5D Planck scale,

$$
M_{5}^{3} \equiv \frac{1}{2 G_{N}^{5 D}} .
$$

The only consistent solution to these equations, satisfying periodicity in $\phi$ and the orbifold parity is illustrated in Fig. 16. But even this solution only exists if the kinks have the right size to reproduce the $\delta$-functions in the equations of motion. This requires the relationships between brane tensions and bulk cosmological constant given by

$$
T^{(1)}=-T^{(2)}=24 k M_{5}^{3} .
$$

Thus the vacuum metric solution is given by

$$
\begin{aligned}
d s^{2} & =\quad e^{-2 k R \phi} \eta_{\mu \nu} d x^{\mu} d x^{\nu}-R^{2} d \phi^{2}, \quad 0 \leq \phi \leq \pi \\
& =e^{-2 k y} \eta_{\mu \nu} d x^{\mu} d x^{\nu}-d y^{2}, \quad 0 \leq y \leq \pi R .
\end{aligned}
$$




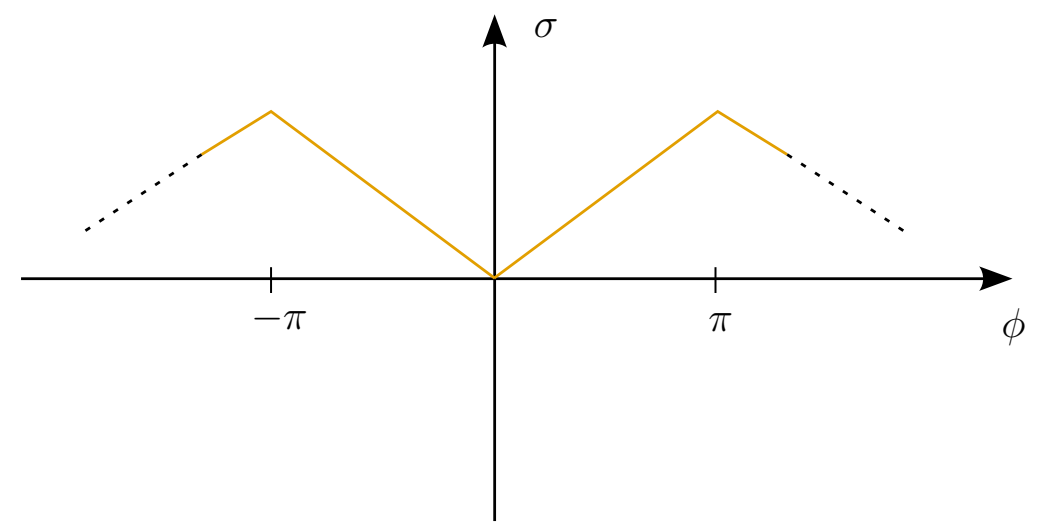

Figure 16: Warp factor solution satisfying orbifold boundary condition

This geometry is a slice of $A d S_{5}, 5 \mathrm{D}$ anti-de Sitter spacetime, which is the maximally symmetric 5D spacetime of negative curvature (just as a sphere is the maximally symmetric space (not spacetime) of positive curvature).

The forms of the massless $4 \mathrm{D}$ modes, the $4 \mathrm{D}$ graviton and radion, are easy to guess:

$$
d s_{(0)}^{2}=e^{-2 k R(x) \phi} g_{\mu \nu}^{(0)}(x) d x^{\mu} d x^{\nu}-R(x)^{2} d \phi^{2}, \quad 0 \leq \phi \leq \pi .
$$

That is we promote the radius, which thusfar has been an integration constant of our vacuum solution, into an $x$-dependent field, and $\eta_{\mu \nu}$ into a dynamical 4-metric, $g_{\mu \nu}^{(0)}(x)$. There is a slick proof that these fluctuations indeed do correspond to the 4D massless modes, that indeed they have no potential at all classically. Plugging the above ansatz into our full action must give a vanishing effective $4 \mathrm{D}$ cosmological term since we found a $4 \mathrm{D}$ Poincare invariant solution. Now a 4D potential for $g_{\mu \nu}^{(0)}$ and $R$ can be computed for $x$ independent fields. A constant linear coordinate transformation can take any non-singular symmetric matrix, $g_{\mu \nu}^{(0)}$, to the form $\eta_{\mu \nu}$. Therefore by residual $4 \mathrm{D}$ general coordinate invariance of the action, plugging $x$-independent $g_{\mu \nu}^{(0)}, R$ must also give zero. Since the effective lagrangian is the negative of the effective potential for constant fields, the effective potential for $g_{\mu \nu}^{(0)}, R$ clearly vanishes. So we have identified the zero-modes and the radion is a modulus again.

Radius stabilization can be readily achieved much as in the unwarped case we studied earlier for the cylindrical spacetime, by adding suitable 5D 
matter. The simplest such implementation is the classical Goldberger-Wise mechanism [21] using a 5D scalar, naturally allowing a moderately large "radius",

$$
k\langle R\rangle \sim \mathcal{O}(10),
$$

where $k(\Lambda)$ is taken not too much smaller than the $5 \mathrm{D}$ effective field theory cutoff. We could also proceed with stabilization by quantum corrections from massive matter as we studied earlier in the unwarped case. We will not pursue these in detail here, just assume some such stabilization, the warping has mild impact on the stabilization.

Ex. Work through Goldberger-Wise stabilization [21].

After radion stabilization, and neglecting the small backreaction of the stabilizing physics on the vacuum metric solution, we have only the $4 \mathrm{D}$ metric zero mode,

$$
d s_{(0)}^{2}=e^{-2 k\langle R\rangle \phi} g_{\mu \nu}^{(0)}(x) d x^{\mu} d x^{\nu}-\langle R\rangle^{2} d \phi^{2}, \quad 0 \leq \phi \leq \pi .
$$

The 4D low-energy effective action for the 4D metric zero mode is obtained by plugging the above ansatz into the fundamental action. The 5D curvature term contains 2-derivative terms, which by 4D Poincare invariance of the vacuum solution must be either two $x$-derivative acting on the zero mode or two $\phi$ derivatives acting on the warp factor. From the 4D point of view all $\phi$ derivatives just contribute to potential terms, which we saw above all cancelled for the 4D graviton zero-mode. So we focus on pairs of $x$-derivatives. Plugging in the zero-mode ansatz, a typical term in the fundamental action has the schematic form,

$$
\begin{aligned}
G_{N}^{5 D} S & \ni \int d^{4} x \int_{0}^{\pi} d \phi \sqrt{G} G^{x x} G^{x x} G^{x x} \partial_{x} G_{x x} \partial_{x} G_{x x} \\
& =\int d^{4} x \int_{0}^{\pi} d \phi R e^{-2 k R \phi} \sqrt{-g^{(0)}} g^{(0) x x} g^{(0) x x} g^{(0) x x} \partial_{x} g_{x x}^{(0)} \partial_{x} g_{x x}^{(0)}
\end{aligned}
$$

which allows us to count powers of the warp factor easily. The residual 4D general coordinate invariance then gives a unique form for the $4 \mathrm{D}$ effective action of the graviton zero-mode,

$$
\begin{aligned}
S_{4 D \text { eff }} & =\frac{1}{G_{N}^{5 D}} \int d^{4} x\left(\int_{0}^{\pi} d \phi R e^{-2 k R \phi}\right) \sqrt{-g^{(0)}} \mathcal{R}_{(4 D)}\left[g^{(0)}\right] \\
& =\frac{1}{2 k G_{N}^{5 D}}\left(1-e^{-2 k \pi R}\right) \int d^{4} x \sqrt{-g^{(0)}} \mathcal{R}_{(4 D)}\left[g^{(0)}\right]
\end{aligned}
$$


That is, at low energies we obtain $4 \mathrm{D}$ General Relativity.

We easily deduce the 4D effective Newton's constant,

$$
16 \pi G_{N}^{4 D \text { eff }}=\frac{2 k G_{N}^{5 D}}{1-e^{-2 k \pi R}} .
$$

Remarkably, even when we take the decompactified limit, $R \rightarrow \infty$, this effective coupling stays finite,

$$
M_{4 P l}^{2}=\frac{M_{5}^{3}}{2 k}\left(1-e^{-2 k \pi R}\right) \underset{R \rightarrow \infty}{\longrightarrow} \frac{M_{5}^{3}}{2 k} .
$$

This is a result of the fact that the zero-mode is localized in the vicinity of the brane at $\phi=0$. This localization mechanism for gravity is sometimes known as the Randall-Sundrum II (RS2) mechanism. Because of gravity localization, the brane at $\phi=0$ is usually called the "Planck brane".

All these classical derivations are considered to take place as the leading approximation of non-renormalizable quantum effective field theory. The control parameter is in general given by

$$
\frac{E^{3}}{M_{5}^{3}} \ll 1
$$

in particular,

$$
\frac{\Lambda^{3 / 5}}{M_{5}^{3}} \sim \frac{\left(M_{5}^{3} k^{2}\right)^{3 / 5}}{M_{5}^{3}} \ll 1 \Longleftrightarrow \frac{k}{M_{5}} \ll 1 .
$$

In practice we usually take all the scales within an order of magnitude or two of the 4D Planck scale,

$$
k \lesssim M_{5} \lesssim M_{4 P l} \sim 10^{18} \mathrm{GeV} .
$$

\section{Warped Hierarchy}

Consider the set-up of Fig. 17, where we have a warped compactification with localized 4D gravity, and now we add a 4D Higgs field by hand (not from $A_{5}$ 's for simplicity), $\delta$-function localized to the opposite brane $(\phi=\pi)$, with $4 \mathrm{D}$ brane-localized action,

$$
S_{\text {Higgs }}=\int d^{4} x \sqrt{-g_{\text {ind }}}\left\{g_{\text {ind }}^{\mu \nu} \partial_{\mu} H^{\dagger} \partial_{\nu} H-\lambda\left(|H|^{2}-v_{0}^{2}\right)^{2}\right\},
$$




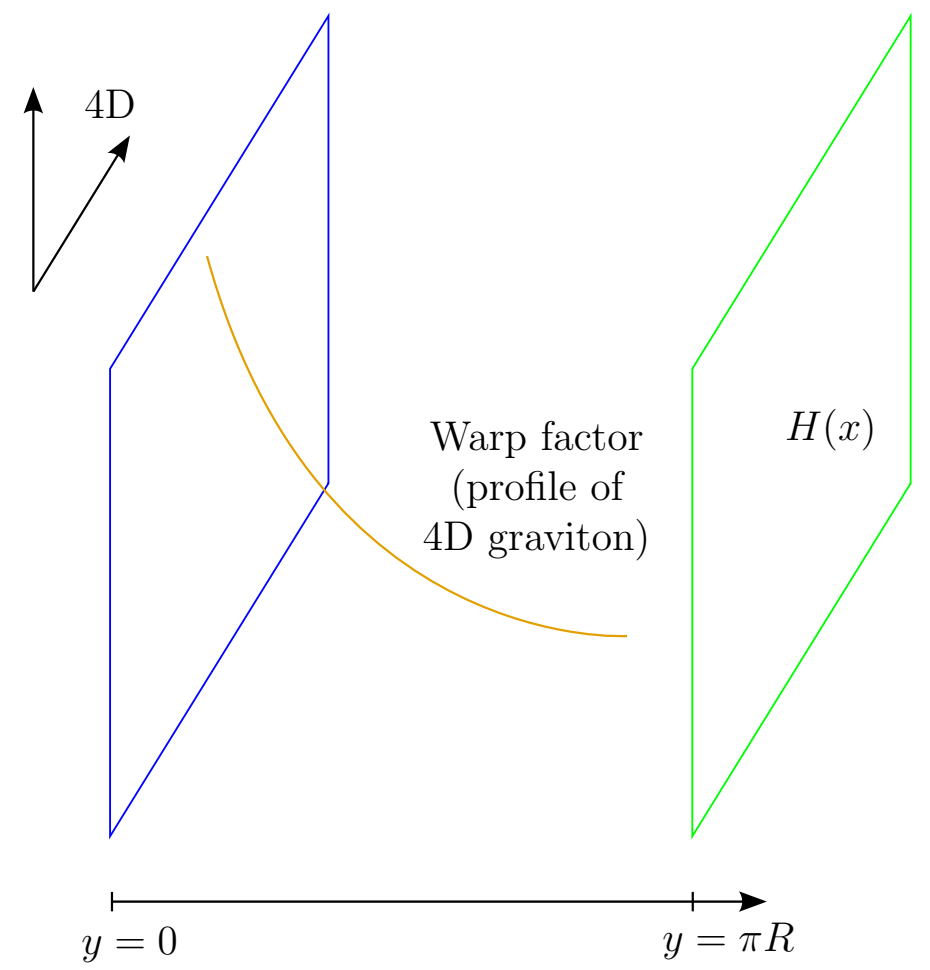

Figure 17: Localization of 4D graviton and Higgs

where

$$
\begin{aligned}
g_{\mu \nu}^{\text {ind }}(x) & =G_{\mu \nu}(x, \phi=\pi) \\
& =e^{-2 k \pi R} g_{\mu \nu}^{(0)}(x),
\end{aligned}
$$

gives the induced $4 \mathrm{D}$ geometry of the brane, and where the second line gives the low-energy approximation where only the gravity zero-mode can propagate. In this approximation the Higgs action becomes

$$
S_{\mathrm{H}}=\int d^{4} x \sqrt{-g^{(0)}}\left\{e^{-2 k \pi R} g_{(0)}^{\mu \nu} \partial_{\mu} H^{\dagger} \partial_{\nu} H-e^{-4 k \pi R} \lambda\left(|H|^{2}-v_{0}^{2}\right)^{2}\right\},
$$

where the warp factor appears like a conventional constant wavefunction renormalization of the Higgs. Canonical normalization of the Higgs is achieved by the field redefinition,

$$
e^{-k \pi R} H \rightarrow H
$$


giving a canonical action,

$$
S_{\mathrm{H}}=\int d^{4} x \sqrt{-g^{(0)}}\left\{g_{(0)}^{\mu \nu} \partial_{\mu} H^{\dagger} \partial_{\nu} H-\lambda(|H|^{2}-\underbrace{e^{-2 k \pi R} v_{0}^{2}})^{2}\right\} .
$$

The miracle is that the "bare" weak scale parameter for the Higgs, $v_{0}$, receives a large warp factor renormalization reducing it to the physical weak scale Higgs VEV,

$$
v=e^{-k \pi R} v_{0} .
$$

Even if $v_{0}$ is very large, Planckian in size, for $k R \sim \mathcal{O}(10)$ one can easily accomodate the physical weak scale, $v \sim 250 \mathrm{GeV}$. Because of this large warping down of UV scales, this brane is sometimes called the IR brane, or in the present context, the "TeV brane".

How does this magic trick work? Consider again the vacuum solution for the $5 \mathrm{D}$ metric,

$$
d s_{\text {vacuum }}^{2}=e^{-2 k y} \eta_{\mu \nu} d x^{\mu} d x^{\nu}-d y^{2} .
$$

In any extra-dimensional locale, $y \sim y_{0}$, we can approximate this metric,

$$
\begin{aligned}
d s_{\mathrm{vac}}^{2} & \sim e^{-2 k y} \eta_{\mu \nu} d x^{\mu} d x^{\nu}-d y^{2} \\
& =\eta_{\mu \nu} d \hat{x}^{\mu} d \hat{x}^{\nu}-d y^{2}
\end{aligned}
$$

which looks just like a piece of 5D Minkowski spacetime provided we define

$$
\hat{x} \equiv e^{-k y_{0}} x, \quad \widehat{m}_{4 D} \equiv e^{k y_{0}} m_{4 D} .
$$

Without warping the translation from 5D to 4D masses is straightforward, so for physics localized in the vicinity of $y_{0}$, we have the translation,

$$
\widehat{m}_{4 D} \sim m_{5 D} \quad \Longrightarrow \quad m_{4 D} \equiv e^{-k y_{0}} m_{5 D} .
$$

For the case of the Higgs,

$$
y_{0}=\pi R
$$

SO

$$
v=e^{-k \pi R} v_{0}
$$

Warped hierarchies are radiatively stable, essentially because even (general coordinate invariant) cutoff scales get warped down near the IR brane, as illustrated in Fig. 18. 


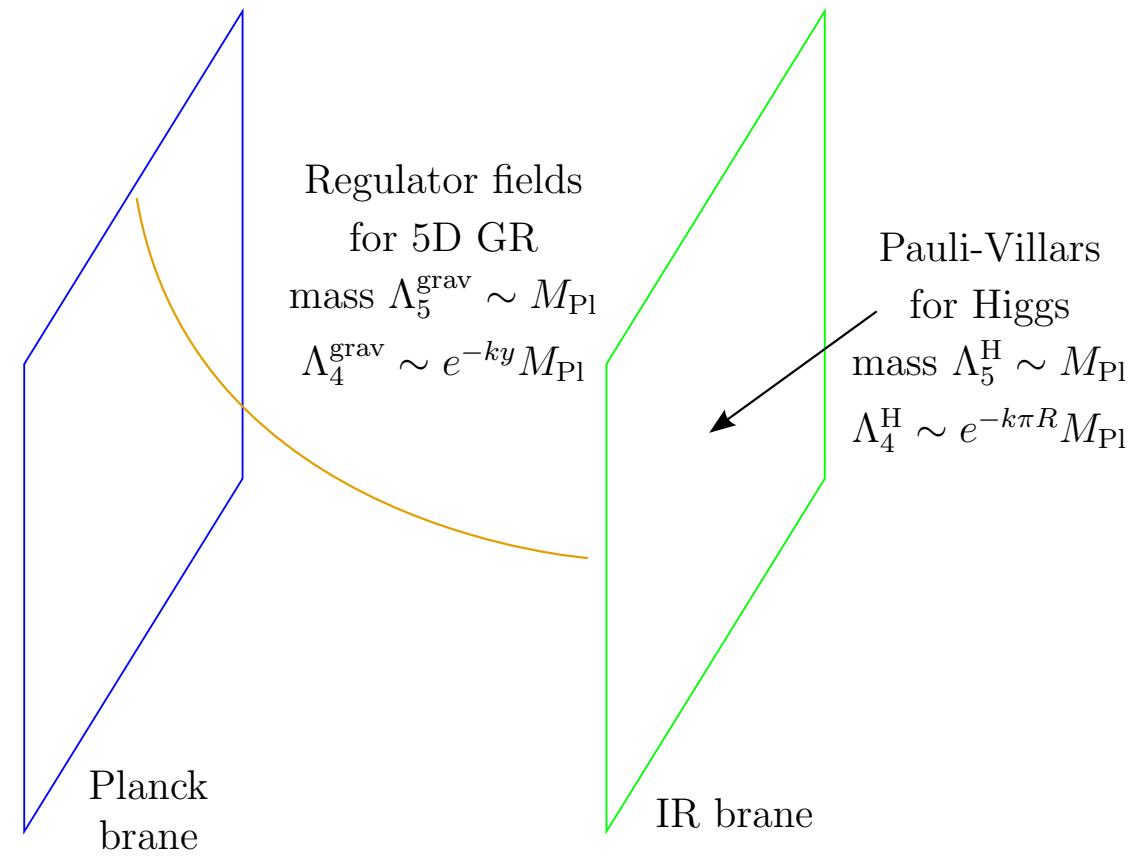

Figure 18: Profiles of 5D regulator fields for gravity and 4D regulator for Higgs

\section{KK Gravitons at Colliders}

Let us begin by removing the IR brane to infinity, $R \rightarrow \infty$, and expand the general 5D metric (in almost axial gauge) about the vacuum solution,

$$
d s^{2}=\left[e^{-2 k|y|} \eta_{\mu \nu}+e^{-k|y| / 2} h_{\mu \nu}(x, y)\right] d x^{\mu} d x^{\nu}-d y^{2} .
$$

The parametrization of fluctuations with the extra exponential is purely for later convenience. It is also convenient to switch to an extra-dimensional coordinate,

$$
z \equiv \operatorname{sgn}(y) \frac{e^{k|y|}-1}{k} .
$$

Plugging the general 5D metric into the fundamental action, and expanding to quadratic order in small fluctuations about the vacuum gives,

$$
G_{N}^{5 D} S=\int d^{4} x \int_{-\infty}^{+\infty} d z\left\{\frac{1}{2} h_{\mu \nu} \square^{\mu \nu \rho \sigma} h_{\rho \sigma}-\frac{1}{2} h_{\mu \nu} \eta^{\mu \nu \rho \sigma} H_{\mathrm{QM}} h_{\rho \sigma}\right\},
$$




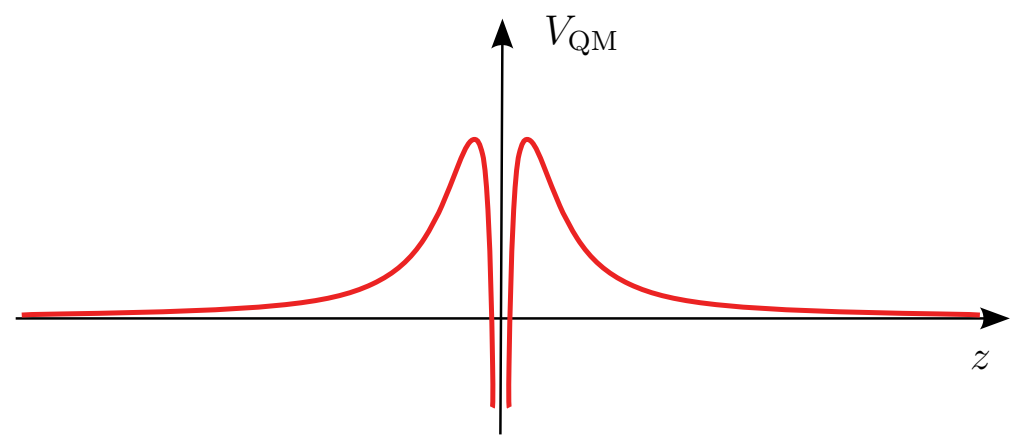

Figure 19: "Volcano" potential of analog quantum mechanics problem describing KK graviton fluctuations

where the first term contains an operator made of two $x$-derivatives while the second term contains an operator made from $y$-derivatives and non-derivative terms, specified by

$$
H_{\mathrm{QM}}=-\frac{1}{2} \partial_{z}^{2}+V_{\mathrm{QM}}(z), \quad V_{\mathrm{QM}}(z) \equiv+\frac{15 k^{2}}{8(k|z|+1)^{2}}-\frac{3 k}{2} \delta(z) .
$$

The unspecified index structures are exactly such that, if $H_{Q M}$ is only a constant and if the fields are purely $4 \mathrm{D}$ fields, and if we get rid of the $z$ integral, this action would just be that of a free massive $4 \mathrm{D}$ spin-2 field, with mass-squared given by the constant $H_{Q M}$. Of course, $H_{Q M}$ is a hermitian operator in the extra dimension, so we should really try to diagonalize the operator. The eigenvalues will then be the 4D mass-squareds of the tower of gravitational KK modes. Note that this eigenvalue problem is analogous to finding the energy eigenvalues of a 1D non-relativistic quantum mechanics problem with "unit" mass and a "volcano potential",

$$
V_{\mathrm{QM}}(z) \equiv+\frac{15 k^{2}}{8(k|z|+1)^{2}}-\frac{3 k}{2} \delta(z),
$$

as illustrated in Fig. 19.

By inspection the analog time-independent Schrodinger equation should have precisely one bound state (which of course must therefore be noneother than our localized zero-mode) and a continuum of waves (asymptoting to sinusoidal waves far away from the volcano) with positive " $E_{Q M}$ " $>0$. Now 
let us put back in the fact that $R<\infty$. In the $z$ coordinates,

$$
\left|z_{\max }\right| \sim e^{k \pi R} / k
$$

While this does little to the bound state mode, it will quantize the previously continuum modes, with

$$
\text { "E } E_{\mathrm{QM}} " \sim\left(n / z_{\max }\right)^{2} .
$$

From this we deduce that the zero mode graviton is accompanied by KK spin-2 excitations with masses given by

$$
m_{\mathrm{KK}}^{2} \sim\left(n k e^{-k \pi R}\right)^{2} .
$$

Even though all the input scales of the fundamental 5D set-up are Planckian, given that the warp factor renormalized the weak scale to its observed size, we get an estimate for KK gravitons,

$$
m_{\mathrm{KK}} \lesssim n M_{P l} e^{-k \pi R} \sim \mathrm{TeV} !
$$

That is, these states are accessible to TeV scale colliders.

Of course it is not good enough to be kinematically accessible, to be seen a new particle must have an appreciable coupling to ordinary matter. If ordinary matter couples to KK gravitons with the same strength as the usual graviton, these states would be completely invisible as a practical matter. Fortunately this is not the case. To be concrete let us assume that all matter, including the Higgs is localized on the TeV brane. They can only couple to the graviton KK modes via their extra-dimensional profiles evaluated on the TeV brane. This determines the relative strength of coupling of different gravity KK modes to brane localized matter since the fundamental coupling constant, the 5D Newton constant, is the same for all the KK modes. The relative strength is easy to estimate. A typical low-lying KK gravity excitation will have roughly a plane-wave wave-function (for the analog non-relativistic quantum mechanics problem) in most of the $z$ space, so that its normalized value on the IR brane is roughly of order $1 / \sqrt{z_{\max }} \sim \sqrt{k} e^{-k \pi R / 2}$. On the other hand, the bound state 4D massless graviton mode has the normalized analog wavefunction evaluated on the IR brane given by $\sim \sqrt{k} e^{-3 k \pi R / 2}$.

Ex. Check this last fact carefully accounting for the factor of $3 / 2$ in the exponent arising from the precise definition of the analog problem in terms of the 5D metric ansatz. 
Thus the amplitude for a KK excited graviton to couple to brane matter is $\sim e^{k \pi R}$ larger than the amplitude for the massless graviton. Now the massless graviton of course couples with $4 \mathrm{D}$ Planck-suppressed $\sim 1 /\left(10^{18} \mathrm{GeV}\right)$ strength which is why such couplings are invisible at $\mathrm{TeV}$ scale colliders. But since we are taking $e^{k \pi R} \sim M_{P l} / \mathrm{TeV}, \mathrm{KK}$ gravitons will couple to matter with only $1 / \mathrm{TeV}$ suppression, that is order one amplitudes at $\mathrm{TeV}$ colliders [22]! In this scenario these exotic spin-2 states should be visible at upcoming colliders such as the LHC. Similar mass splitting, wavefunctions and coupling strengths to IR brane matter hold for KK excitations of any field (with any spin) one considers in the 5D bulk.

\section{Warped (Fermionic) Bulk Matter}

A useful and pioneering reference for this section and the next is Ref. 23. Let us consider $5 \mathrm{D}$ fermions in the warped context subject to the orbifold boundary conditions,

$$
\begin{aligned}
& P\left(\Psi_{\mathrm{L}}\right)=+\Psi_{\mathrm{L}} \\
& P\left(\Psi_{\mathrm{R}}\right)=-\Psi_{\mathrm{R}} .
\end{aligned}
$$

Using the proper-distance $y$ coordinate ranging from $-\pi R$ to $\pi R$, we consider a bulk action of the form

$$
S_{\Psi}=\int d^{4} x \int_{-\pi R}^{+\pi R} d y e^{-4 k|y|} \bar{\Psi}\left(i \Gamma^{M} D_{M}-m \operatorname{sgn}(y)\right) \Psi .
$$

In general, the covariant derivative for fermions cannot be written directly in terms of the metric alone, but requires a compatible "spin-connnection". We will just consider the RS1 vacuuum metric for which the compatible covariant derivatives are given by

$$
\begin{aligned}
D_{\mu} & =\partial_{\mu}-\frac{1}{2} \Gamma_{5} \Gamma_{\mu} k \operatorname{sgn}(y) \\
D_{5} & =\partial_{y} .
\end{aligned}
$$

The exponential is just the measure factor arising from the root of the metric determinant as usual, but it appears that the mass term has explicit $y$ dependence in order to be compatible with the orbifold parity symmetry. This 
is not really cheating, such a mass term can be thought of as arising from a Yukawa coupling to a orbifold-parity-odd 5D scalar field, whose fluctuations are very massive but whose VEV is non-zero and proportional to $\operatorname{sgn}(y)$. The curved space $\Gamma^{N}$ are given in terms of the usual flat space Dirac matrices by $\Gamma_{\mu}=e^{-k|y|} \gamma_{\mu}, \Gamma_{5}=-i \gamma_{5}, \Gamma^{\mu}=e^{+k|y|} \gamma^{\mu}, \Gamma^{5}=i \gamma_{5}$.

Decomposing the action in $4 \mathrm{D}$ notation, and making the convenient field redefinition, $\Psi \equiv e^{+3 / 2 k|y|} \hat{\Psi}$

$$
S_{\Psi}=\int d^{4} x \int d y \hat{\bar{\Psi}}\left\{i \not \partial+e^{-k|y|}\left[\frac{k}{2} \operatorname{sgn}(y) \gamma_{5}-\gamma_{5} \partial_{y}-m \operatorname{sgn}(y)\right]\right\} \hat{\Psi}
$$

Despite the 5D mass parameter, which we will from now on dimensionlessly parametrize as

$$
c \equiv \frac{m}{k}
$$

there are 4D massless chiral fermion zero-modes. We clearly get a zero-mode from the equation of motion if the mode is annihilated by the 4D Dirac operator, so that

$$
\left[k\left(\frac{\gamma_{5}}{2}-c\right) \operatorname{sgn}(y)-\gamma_{5} \partial_{y}\right] \hat{\Psi}=0
$$

We see two possible chiral solutions to this equation. The left-handed one,

$$
\hat{\Psi}_{\mathrm{L}}(x, y)=\hat{\Psi}_{\mathrm{L}}^{(0)}(x) e^{\left(\frac{1}{2}-c\right) k|y|},
$$

satisfies the orbifold parity condition and is therefore physical. The righthanded one,

$$
\hat{\Psi}_{\mathrm{R}}(x, y)=\hat{\Psi}_{\mathrm{R}}^{(0)}(x) e^{\left(\frac{1}{2}+c\right) k|y|},
$$

does not satisfy being parity-odd, and therefore is inadmissible. The parity therefore gives us a chiral 4D massless left-handed zero-mode.

While the 5D mass parameter, $c$, does not affect the existence of $4 \mathrm{D}$ chiral modes, they clearly influence their profile in the extra dimension, as illustrated in Fig. 20. If the Higgs is considered to be localized on the IR brane still, 4D Yukawa couplings with two species of chiral zero modes coming from bulk fermions with mass parameters $c_{a}, c_{b}$ will be given by

$$
\underset{\text { couplings }}{4 D \text { Yukawa }} \sim e^{\left(\frac{1}{2}-c_{a}\right) k \pi R} e^{\left(\frac{1}{2}-c_{b}\right) k \pi R} \times \begin{gathered}
5 D \text { Yukawa } \\
\text { couplings }
\end{gathered} .
$$




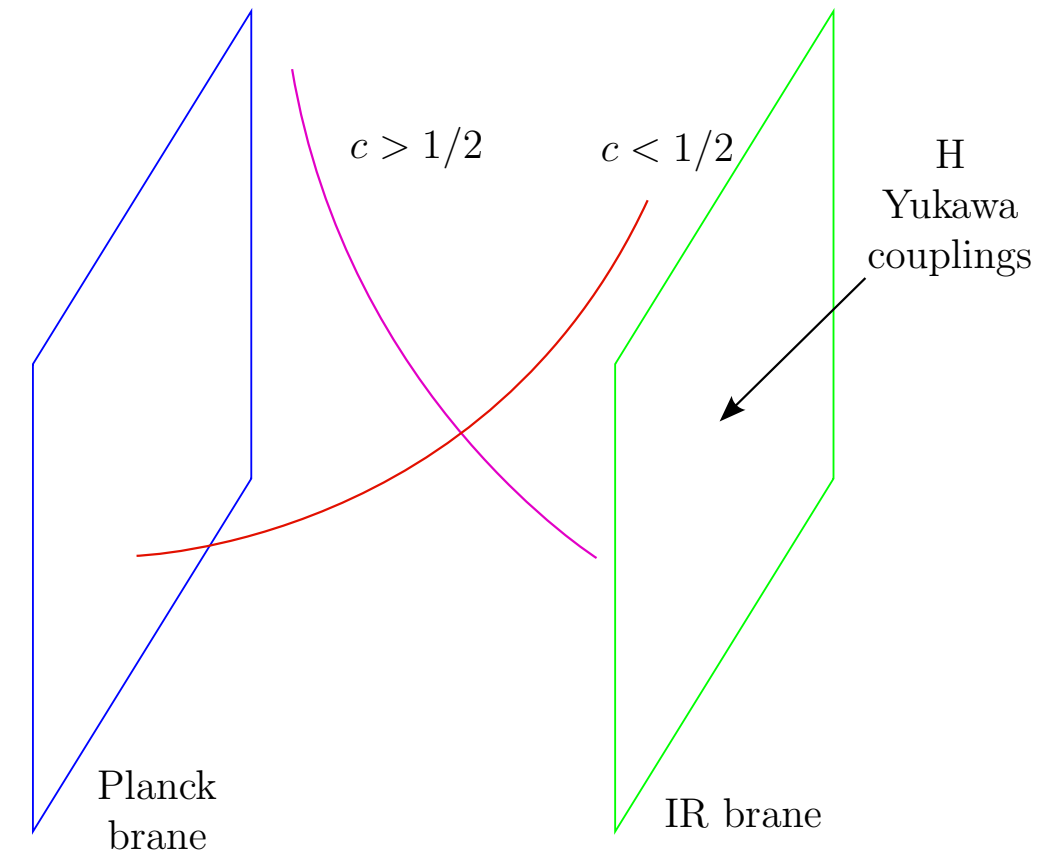

Figure 20: Fermion zero-mode profiles for different 5D fermion masses

Thus even without large hierarchies at the 5D level, hierarchical effective Yukawa couplings are naturally generated. In the real world, we can identify light fermions as chiral modes arising from bulk fermions with $c>1 / 2$, and heavy fermions with chiral modes arising from bulk fermions with $c<$ $1 / 2$. Therefore light fermion profiles are suppressed at the IR brane. This suppresses their wave-function overlap with low-lying KK excitations of all bulk fields, thereby suppressing a host of dangerous KK-mediated effects. This is the central part of an automatic GIM mechanism suppressing flavorchanging neutral currents. On the other hand one can predict that the heavy top quark in this scenario should display significant non-standard corrections to its couplings. 


\section{Warped Bulk YM}

Of course if fermionic matter lives in the 5D bulk so must the gauge fields. The 5D YM action in background curved space is given by

$$
S=-\frac{1}{4} \int d^{4} x \int_{-\pi}^{+\pi} d \phi \sqrt{G} G^{M N} G^{K L} F_{M K} F_{N L}
$$

We will consider the RS warped vacuum background.

We begin by considering the orbifold boundary conditions given by

$$
\begin{aligned}
P\left(A_{\mu}\right) & =+A_{\mu} \\
P\left(A_{5}\right) & =-A_{5} .
\end{aligned}
$$

In almost axial gauge, the single expected $A_{5}$ mode is eliminated by the orbifold conditions, but $A_{\mu}$ does give rise to a zero-mode.

Ex. Show that

$$
A_{\mu}(x, \phi)=A_{\mu}^{(0)}(x)
$$

is the zero-mode $4 \mathrm{D}$ gauge boson and that if coupled to matter (or if nonabelian), the effective 4D gauge coupling is given by

$$
g_{4}=\frac{g_{5}}{\sqrt{2 \pi R}} .
$$

Note the form of the zero-mode and the 4D coupling look exactly as they did without warping. Gauge theory is very different from gravity in this regard. In particular the warped explanation for "why gravity is weak" (the zero-mode graviton of course) does not simultaneously greatly weaken the strength of gauge forces, which is obviously a good thing.

The other interesting example to consider is given by orbifold conditions,

$$
\begin{aligned}
& P\left(A_{\mu}\right)=-A_{\mu} \\
& P\left(A_{5}\right)=+A_{5},
\end{aligned}
$$

so that now there is a $4 \mathrm{D}$ scalar zero-mode but not a vector zero-mode. (Of course, as illustrated earlier in flat spacetime, for non-abelian gauge group different gauge fields corresponding to different generators can be treated with different orbifold boundary conditions. We will not consider that level of 
complexity here.) To isolate the scalar zero-mode in the warped background we first look at all terms in the action containing $A_{5}$,

$$
\begin{aligned}
S & \ni \frac{1}{2} \int d^{4} x \int d \phi R e^{-4 k|\phi| R} e^{2 k|\phi| R}\left(\partial_{5} A_{\mu}-\partial_{\mu} A_{5}\right)^{2} \\
& \ni \frac{1}{2} \int d^{4} x \int d \phi R e^{-2 k|\phi| R}\left[\left(\partial_{\mu} A_{5}\right)^{2}-2 \partial_{5} A_{\mu} \partial^{\mu} A_{5}\right] .
\end{aligned}
$$

The usual almost axial gauge does not disentangle the vector-scalar mixing in warped spacetime and is therefore not convenient. But if we try

$$
A_{5}(x, \phi)=A_{5}^{(0)}(x) e^{+2 k|\phi| R},
$$

the mixing term vanishes (as you can check by doing an integration by parts with respect to the $\phi$ derivative). The $4 \mathrm{D}$ effective action for the zero-mode scalar below the mass of the lightest KK excitations $\left(\sim k e^{-k \pi R}\right)$ is then given by

$$
\begin{aligned}
S_{\mathrm{eff} 4 D} & =\frac{1}{2} \int d^{4} x \int_{-\pi}^{+\pi} d \phi R e^{+2 k|\phi| R}\left(\partial_{\mu} A_{5}^{(0)}\right)^{2} \\
& =\int d^{4} x \frac{e^{2 k \pi R}-1}{2 k}\left(\partial_{\mu} A_{5}^{(0)}\right)^{2} .
\end{aligned}
$$

As the zero-mode ansatz shows such massless scalars are localized (but not $\delta$-function localized) near the IR brane and therefore makes a good Higgs candidate 24]!

One can ask what the advantage is of having the Higgs realized as the fifth component of a gauge field as opposed to as a fundamental IR-branelocalized scalar. The answer is that in the former case radiative corrections to the scalar mass-squared are effectively cut off at $\sim m_{\mathrm{KK}} \sim k e^{-k \pi R}$ while in the latter case the cutoff is the "warped down" fundamental cutoff $\Lambda_{\mathrm{UV}} e^{-k \pi R}$, which is parametrically larger. This is a generalization of the flat spacetime result we derived in detail earlier where the cutoff on the scalar mass was effectively $m_{\mathrm{KK}} \sim 1 / R$. One can repeat the kind of calculations we did at the beginning of these lectures which carve out an $A_{5}$ effective potential, but now in the warped context, to build realistic warped models of the Higgs and electroweak symmetry breaking. The central considerations needed to achieve realism are worked out in Ref. 25]. Combining these with the Higgs realized as an $A_{5}$ was done in Ref. [26]. 


\section{Last Words}

Well, I have just gone through the simplest examples of many of the interesting mechanisms connected with extra dimensions. They can appear together in interesting combinations. When this happens there is greater complexity

and the probability for error is multiplied. I have found that in the arena of warped compactifications, the qualitative insight gained from the AdS/CFT connection between such compactifications and strongly coupled 4D dynamics, has saved me time and time again from errors. It's like "checking units" as an undergraduate, in principle it's not necessary, but in practice indispensible. But while checking units is dull, the AdS/CFT connection is pure magic! It also allows you to design more efficient methods of analysing your models quantitatively. You can learn to think in terms of this connection by starting with Refs. 7] [9].

\section{Acknowledgements}

The author is supported by NSF grant P420-D36-2043-4350. He is grateful to Dmitry Belyaev for assistance with the figures and equations, and for catching several errors.

\section{References}

[1] C. Csaki, arXiv:hep-ph/0404096.

[2] I. Z. Rothstein, arXiv:hep-ph/0308266.

[3] R. Rattazzi, Cargese lectures in "Particle Physics and Cosmology: The Interface", eds. D. Kazakov and G. Smadja, Springer 2005

[4] M. A. Luty, arXiv:hep-th/0509029

[5] H. Ooguri and Z. Yin, arXiv:hep-th/9612254.

[6] J. Polchinski, "String Theory" Vol. 1, Ch.8, Cambridge University Press (1998).

[7] J. M. Maldacena, arXiv:hep-th/0309246. 
[8] O. Aharony, S. S. Gubser, J. M. Maldacena, H. Ooguri and Y. Oz, Phys. Rept. 323, 183 (2000) arXiv:hep-th/9905111.

[9] N. Arkani-Hamed, M. Porrati and L. Randall, JHEP 0108, 017 (2001) arXiv:hep-th/0012148|; R. Rattazzi and A. Zaffaroni, JHEP 0104, 021 (2001) arXiv:hep-th/0012248.

[10] N. Arkani-Hamed, S. Dimopoulos and G. R. Dvali, Phys. Lett. B 429, 263 (1998) arXiv:hep-ph/9803315.

[11] N. S. Manton, "A New Six-Dimensional Approach To The WeinbergSalam Model," Nucl. Phys. B 158, 141 (1979); D. B. Fairlie, Phys. Lett. B 82, 97 (1979); J. Phys. G 5, L55 (1979); P. Forgacs and N. S. Manton, Commun. Math. Phys. 72, 15 (1980); S. Randjbar-Daemi, A. Salam and J. A. Strathdee, Nucl. Phys. B 214, 491 (1983).

[12] Y. Hosotani, Phys. Lett. B 126, 309 (1983); Annals Phys. 190, 233 (1989).

[13] M. Quiros, arXiv:hep-ph/0302189.

[14] C. A. Scrucca, M. Serone and L. Silvestrini, Nucl. Phys. B 669, 128 (2003) arXiv:hep-ph/0304220.

[15] H. Georgi, "Weak Interactions and Modern Particle Theory", Benjamin/Cummings, Menlo Park (1984).

[16] Z. Chacko, M. A. Luty and E. Ponton, JHEP 0007, 036 (2000) arXiv:hep-ph/9909248.

[17] S. Weinberg, Rev. Mod. Phys. 61, 1 (1989).

[18] L. Randall and R. Sundrum, Phys. Rev. Lett. 83, 3370 (1999) arXiv:hep-ph/9905221.

[19] L. Randall and R. Sundrum, Phys. Rev. Lett. 83, 4690 (1999) arXiv:hep-th/9906064.

[20] H. Georgi, A. K. Grant and G. Hailu, Phys. Lett. B 506, 207 (2001) arXiv:hep-ph/0012379. 
[21] W. D. Goldberger and M. B. Wise, Phys. Rev. Lett. 83, 4922 (1999) arXiv:hep-ph/9907447.

[22] H. Davoudiasl, J. L. Hewett and T. G. Rizzo, Phys. Rev. Lett. 84, 2080 (2000) arXiv:hep-ph/9909255.

[23] T. Gherghetta and A. Pomarol, Nucl. Phys. B 586, 141 (2000) arXiv:hep-ph/0003129.

[24] R. Contino, Y. Nomura and A. Pomarol, Nucl. Phys. B 671, 148 (2003) arXiv:hep-ph/0306259.

[25] K. Agashe, A. Delgado, M. J. May and R. Sundrum, JHEP 0308, 050 (2003) arXiv:hep-ph/0308036.

[26] K. Agashe, R. Contino and A. Pomarol, Nucl. Phys. B 719, 165 (2005) arXiv:hep-ph/0412089. 